\title{
Identification and Analysis of microRNAs Responsive to Abscisic Acid and Methyl Jasmonate Treatments in Persicaria minor
}

(Pengenalpastian dan Analisis Gerak Balas mikroRNA kepada Rawatan Asid Absisik dan Metil Jasmonat dalam Persicaria minor)

Abdul FATAh A. SAMAD, NAZARUdDin NAZARUddin, JAEYRES JANi \& ISMANIZAN ISMAiL*

ABSTRACT

Persicaria minor has been recognised as a plant with high content of volatile organic compounds (VOC) especially terpenoid and green leaf volatile (GLV). Previous finding had showed signaling molecules such as abscisic acid (ABA) and methyl jasmonate (MeJA) can increase the VOC content in plant. In this study, we performed next generation sequencing (NGS) of small RNA to uncover miRNAs roles and their response to both phytohormones (ABA and MeJA) in P. minor. For both ABA and MeJA treated P. minor, small RNA libraries containing 17,253,566 and 40,437,576 reads were generated, respectively. In addition, 18,634,904 reads were generated in plant treated with sterile distilled water which served as control. In these libraries, a total of 88 miRNAs were identified, comprising 41 known and 47 novel miRNAs. It was observed that 21 and 38 miRNAs were significantly regulated in ABA and MeJA libraries, respectively. Four selected miRNAs related to VOC pathways were subjected to RT-qPCR analysis and found to display diverse expression patterns with their targets. This study provides the initial framework for further exploration of miRNA roles in ABA and MeJA responses.

Keywords: Abscisic acid; methyl jasmonate; microRNA; Persicaria minor; volatile organic compound

\section{ABSTRAK}

Persicaria minor telah dikenal pasti sebagai tumbuhan yang mempunyai kandungan sebatian organik meruap (VOC) yang tinggi terutama terpenoid dan sebatian daun hijau meruap (GLV). Kajian lepas menunjukkan molekul pengisyaratan seperti asid absisik (ABA) dan metil jasmonat (MeJA) boleh meningkatkan kandungan VOC dalam tumbuhan. Dalam kajian ini, kami menjalankan penjujukan generasi terkini (NGS) RNA kecil untuk merungkai peranan miRNA dan tindak balasnya terhadap kedua-dua fitohormon (ABA dan MeJA) dalam P. minor. Bagi kedua-dua rawatan ABA dan MeJA terhadap P. minor, perpustakaan kecil RNA masing-masing telah menjana sejumlah 17,253,566 dan 40,437,576 bacaan. Tambahan lagi, sejumlah 18,634,904 bacaan telah dijana daripada tumbuhan terawat air suling steril yang bertindak sebagai kawalan. Dalam perpustakaan tersebut, sejumlah 88 miRNA telah dikenal pasti yang terdiri daripada 41 miRNA yang telah diketahui fungsinya dan 47 miRNA novel. Sejumlah 21 dan 38 miRNA masingmasing telah dicerap dikawal atur secara signifikan dalam perpustakaan ABA dan MeJA. Sebanyak empat miRNA yang berkait dengan tapak jalan VOC telah dikaji melalui analisis RT-qPCR dan didapati menunjukkan corak pengekspresan yang pelbagai terhadap transkrip sasaran masing-masing. Kajian ini menyediakan rangka kerja awal untuk penerokaan selanjutnya mengenai peranan miRNA dalam tindak balas ABA dan MeJA.

Kata kunci: Asid absisik; metil jasmonat; mikroRNA; Persicaria minor; sebatian organik meruap

\section{INTRODUCTION}

Persicaria minor or known as 'kesum' is a medicinal plant with high content of secondary metabolites (Ee et al. 2014). These secondary metabolites are responsible for its pharmaceutical properties, such as its antioxidant, antiviral, antifungal, antiulcer and antimicrobial activities (Christapher et al. 2015). Additionally, due to its unique aroma, this plant is commonly used as food additives in local dishes in Southeast Asia countries (Christapher et al. 2015). Among these secondary metabolites, flavonoid and terpenoid were dominant (Baharum et al. 2010;
Roslan et al. 2012). For example, $\beta$-caryophyllene is the highest terpenoid compound in P. minor essential oil (Baharum et al. 2010). In addition, other volatile compounds were also detected in $P$. minor for example decanal and dodecanal which belong to aliphatic aldehyde group (Christapher et al. 2015).

Phytohormones are signaling molecules which are essential in regulating plant growth and stress responses. In addition, their ability to act as a messenger in plant cell make them suitable candidates for mediating biosynthesis of particular product (Liang et al. 2013). $\mathrm{ABA}$ is a recognised elicitor that induces plant secondary 
metabolite. Previously, ABA treatments on Salvia miltiorrhiza have led to the highlevel production of the active compound, tashinones (Yang et al. 2012). Similarly, jasmonic acid or its derivatives, methyl jasmonate (MeJA) participates in a variety of growth processes, stress response and secondary metabolite induction (Yan \& Xie 2015). For instance, exogenous application of MeJA enhanced taxol formation in Taxus cuspidata suspension culture (Lenka et al. 2015). Based on previous study, both phytohormones, ABA and MeJA were able to alter gene expression which leads to the production of a particular compound at the downstream level.

Gene expressions are coordinated through multilayers level, beginning at epigenetic, transcriptional and post-transcriptional levels to ensure precise control. At post-transcriptional level, a group of small RNA, miRNA, is known to be involved in various biological processes in plant (Samad et al. 2017). miRNA acts as gene silencer by binding to the target gene to induce cleavage or translational inhibition (Samad et al. 2017). Latest miRBase version (version 22) showed a total of 38,589 miRNA that had been discovered in animals, plants and viruses, and the number is expected to be increasing in the future (Kozomara et al. 2019). This is an indicator that miRNA has already gained researchers attention due to its regulatory role and subsequently recognised as potential tool for manipulating gene expression to produce plant with desirable traits. Furthermore, the public database will facilitate the discovery of miRNA in other plant species especially for plant with no genome information available.

To date, several approaches had been carried out at transcriptional level to explore the elicitation effect of MeJA towards $P$. minor. Those approaches include construction of subtracted cDNA library and transcriptomic library. Among the induced genes were peroxidase and defense related genes (Gor et al. 2011; Rahnamaie-Tajadod et al. 2017). However, at present, not much information is known about the post-transcriptional regulation in $P$. minor represented by miRNA. Hence, this study focused on characterisation of miRNA and their response in $P$. minor under ABA and MeJA treatments.

\section{MATERIALS AND METHODS}

\section{PLANT MATERIALS AND TREATMENTS}

P. minor plants were grown and propagated in controlled condition at Rumah Tumbuhan, Universiti Kebangsaan Malaysia. Approximately, 6 weeks old plants were selected for MeJA and ABA treatments. The treatments were carried out as mentioned in previous report (Nazaruddin et al. 2017). Two sets of $P$. minor plants were sprayed with $100 \mu \mathrm{M}$ of MeJA and $100 \mu \mathrm{M}$ of ABA, while the control plants were sprayed with distilled water. Two biological replicates were prepared for each treatment. For MeJA-treated plants, leaf samples were harvested after 2 days while ABA-treated plants were harvested after 3 days of treatment. These periods of treatments were selected based on the changes in leaf morphology of the P. minor. Prior to RNA extraction, $P$. minor leaves were harvested and immediately stored in $-80{ }^{\circ} \mathrm{C}$ freezer for further use.

\section{TOTAL RNA EXTRACTION AND SMALL RNA LIBRARY CONSTRUCTION}

Approximately $0.1 \mathrm{~g}$ of leaves were ground to extract total RNA from mock-inoculated (K) leaves, and ABA and MeJA treated leaves using PureLink ${ }^{\circledR}$ Plant RNA reagent (Invitrogen, USA) according to the manufacturer's protocol. The RNA integrity number (RIN) from each sample was measured using Nanodrop 1000 (ThermoFisher Scientific Inc., USA), gel electrophoresis and Agilent 2100 Bioanalyzer (Agilent Technology, USA). Total RNA with RIN of at least 7 was selected for small RNA library construction. Then, the small RNA libraries were sequenced using Ilumina platform (HiSeq 2500) in Rapid Run mode.

\section{DIFFERENTIAL GENE EXPRESSION}

Prior to identification of differentially expressed miRNA, the data from each library was normalised to transcript per million (TPM). The analysis was carried out using Baggerley's test from CLC Genomics software (Baggerly et al. 2003). A threshold of a P-value $<0.05$ and a fold-change $\geq 2$ were used to determine significant changes of miRNA expression (Audic \& Claverie 1997). Additionally, the false discovery rate (FDR $<0.05$ ) correction method was deployed to correct the P-value which then referred to determine the significantly expressed miRNA (Benjamini \& Hochberg 1995). Transcriptomic sequence was retrieved from GeneBank under accession number SRX669305 (Loke et al. 2016).

\section{PREDICTION OF PUTATIVE NOVEL MIRNA}

Novel miRNA identification was carried out using homology search of unannotated small RNA sequences against $P$. minor transcriptomes. The potential transcript was investigated based on the ability of the sequence to form secondary structure and value of Minimum Folding Energy Index (MFEI) (Zhang et al. 2006). Sequence folding was carried out using mFold software (http:// unafold.rna.albany.edu/) (Markham \& Zuker 2008). The parameters for determination of MFEI were described in previous report (Samad et al. 2018).

\section{miRNA TARGET PREDICTION AND GENE ONTOLOGY ENRICHMENT}

PsRobot(http://omicslab.genetics.ac.cn/psRobot/) was employed to predict the target for miRNA (Wu et al. 2012). Since $P$. minor genome is still not available, previous transcriptomic library was used in this analysis. This analysis used overall score 4.0 to allow more detection of miRNA targets. In addition, gene ontology analysis was 
carried out using WEGO software (http://wego.genomics. org.cn/) (Ye et al. 2018).

\section{EXPRESSION ANALYSIS USING RT-QPCR}

cDNA for each sample was synthesised using RevertAid Reverse Transcriptase (Thermofisher, USA) according to the manufacturer's protocols. RT-qPCR analysis for ABA and MeJA treated samples were carried out in series of timeline for three consecutive days. A set of mock treated plants with sterile distilled water were prepared as control (Day 0). The RT-qPCR was carried out using
Thermo Scientific Maxima SYBR Green qPCR Master Mix (Thermofisher, USA). miRNA mature sequence was used as miRNA forward primer (Table 1) and universal primer from miScript SYBR ${ }^{\circledR}$ Green PCR Kit (Qiagen, Germany) was used as reverse primer. PrimerQuest Tool Integrated DNA Technologies (https://sg.idtdna.com/) was used to design forward and reverse primers for target genes (Table 2). For reference genes, 5.8s rRNA was used for miRNA and tubulin was used for target genes. Relative gene expression was analysed and calculated according to Livak and Schmittgen (2001).

TABLE 1. List of miRNA primers

\begin{tabular}{lc}
\hline \multicolumn{1}{c}{ miRNA } & Primer sequence \\
\hline pmi-miR396a & 5'-GTT CAA TAA AGC TGT GGG A-3' \\
pmi-miR396b & 5'-GGG GTT CAA TAA AGC TGT TGG AA-3' \\
pmi-miR6173 & 5'-GGG GGA GCC GTA AAC GAT GGA TA-3' \\
pmi-miR6300 & 5'-GGG GGT CGT TGT AGT ATA GTG GA-3' \\
pmi-miRNew-27 & 5'-CGT GTT ATC GTG TCG GAT A-3' \\
\hline
\end{tabular}

TABLE 2. List of target primers

\begin{tabular}{lc}
\hline \multicolumn{1}{c}{ Target genes } & Primer sequence \\
\hline \multirow{2}{*}{ Peroxidase } & 5'-GGA ACC CAA ACC ACA ACT TTC-3' (Forward) \\
& 5'-CTG TCG CCA ATC TTT CAT CAA TC-3' (Reverse) \\
ADH1 & 5'-TAC TTGTTC AGC AAA TCT CTC CA-3' (Forward) \\
& 5'-CTC TTC AGG TTG ATG TGT CCT T-3' (Reverse) \\
Sesquiterpene synthase & 5'-AGA CGT AGT GAG CAA CCA AC-3' (Forward) \\
& 5'-CTT GGC ATA CCC TTG TGG TAA-3' (Reverse) \\
HMGR & 5'-GCC AAC ATT GTG TCT GCT ATC-3' (Forward) \\
& 5'-ATG GTC ACG GAG ATG TGA AG-3' (Reverse)
\end{tabular}

\section{RESULTS AND DISCUSSION}

DEEP SEQUENCING ANALYSIS OF SMALL RNA

To investigate the miRNAs that had responded to $A B A$ and MeJA treatments, three types of small RNA libraries (K, ABA, and MeJA) were constructed. The high-throughput sequencing generated around 18,634,904, 17,253,566, and 40,437,576 reads in three libraries, respectively. After removing adaptor sequences, low quality reads and filtering sequences into 18-30 nt, K, $\mathrm{ABA}$ and MeJA libraries produced 10,973,180, 11,571,770 and $21,458,916$ sequences, respectively. The annotation and statistics of $P$. minor small RNAs was documented in Table 3 . 
TABLE 3. Statistics of small RNA in K, ABA and MeJA libraries

\begin{tabular}{|c|c|c|c|c|}
\hline & Total reads & Percent (\%) & Unique reads & Percent (\%) \\
\hline \multicolumn{5}{|l|}{ K library } \\
\hline \multirow[t]{2}{*}{ Raw reads } & $18,634,905 \pm$ & & & \\
\hline & $10,481,749$ & & & \\
\hline \multirow[t]{2}{*}{ Clean reads (18-30nt) } & $10,973,181 \pm$ & 100.0 & $1,852,647 \pm$ & 100.0 \\
\hline & $6,171,438$ & & 931,365 & \\
\hline miRNA & $28,193 \pm 9,808$ & 0.26 & $1,124 \pm 600$ & 0.06 \\
\hline \multirow[t]{2}{*}{ Rfam } & $694,910 \pm$ & 6.33 & $84,125 \pm$ & 4.54 \\
\hline & 309,740 & & 13,931 & \\
\hline Unannotated & $\begin{array}{c}10,250,078 \pm \\
5,871,505\end{array}$ & 93.41 & $\begin{array}{c}1,767,398 \pm \\
918,034\end{array}$ & 95.40 \\
\hline \multicolumn{5}{|l|}{ ABA library } \\
\hline \multirow[t]{2}{*}{ Raw reads } & $17,253,566 \pm$ & & & \\
\hline & $18,826,895$ & & & \\
\hline \multirow[t]{2}{*}{ Clean reads (18-30nt) } & $11,571,771 \pm$ & 100.0 & $1,580,735 \pm$ & 100 \\
\hline & $12,886,302$ & & 153,3613 & \\
\hline \multirow[t]{2}{*}{ miRNA } & $22,049 \pm$ & 0.19 & $538 \pm$ & 0.03 \\
\hline & 26,830 & & 434 & \\
\hline \multirow[t]{2}{*}{ Rfam } & $579,375 \pm$ & 5.00 & $68,857 \pm$ & 4.36 \\
\hline & 624,828 & & 45,657 & \\
\hline \multirow[t]{2}{*}{ Unannotated } & $10,970,347 \pm$ & 94.8 & $1,511,349 \pm$ & 95.61 \\
\hline & $12,234,644$ & & $1,487,522$ & \\
\hline \multicolumn{5}{|l|}{ MeJA library } \\
\hline \multirow[t]{2}{*}{ Raw reads } & $40,437,576$ & & & \\
\hline & $\pm 9,816,458$ & & & \\
\hline \multirow[t]{2}{*}{ Clean reads (18-30nt) } & $21,458,917 \pm$ & 100.0 & $2,163,212 \pm$ & 100.0 \\
\hline & $3,343,499$ & & 339,378 & \\
\hline \multirow[t]{2}{*}{ miRNA } & $143,282 \pm$ & 0.67 & $2,773 \pm 179$ & 0.13 \\
\hline & 51,799 & & & \\
\hline \multirow[t]{2}{*}{ Rfam } & $1,089,945 \pm$ & 5.08 & $119,792 \pm$ & 5.54 \\
\hline & 21,209 & & 12,669 & \\
\hline \multirow[t]{2}{*}{ Unannotated } & $20,225,690$ & 94.25 & $2,040,647$ & 94.33 \\
\hline & $\pm 3,374,089$ & & $\pm 326,888$ & \\
\hline
\end{tabular}


The results showed that $28,193(0.26 \%), 22,049$ $(0.19 \%)$, and $143,282(0.67 \%)$ of miRNA were discovered in $\mathrm{K}, \mathrm{ABA}$ and MeJA libraries, respectively. In addition, for $\mathrm{K}$ and ABA libraries, small RNAs with $22 \mathrm{nt}$ in length were most abundant while small RNA with 20 nt in length was most abundant in MeJA library (Figure 1). Previous study showed that small RNAs with $21 \mathrm{nt}$ in length was the most abundant miRNA in $A$. thaliana (Pontes et al. 2009). Around 694,910 (6.33\%), 579,375 (5.00\%) and 1,089,945 (5.08\%) sequences were mapped against Rfam database in $\mathrm{K}, \mathrm{ABA}$ and MeJA libraries, respectively. The rest of the unmapped sequences were used to find the potential novel miRNA in P. minor.

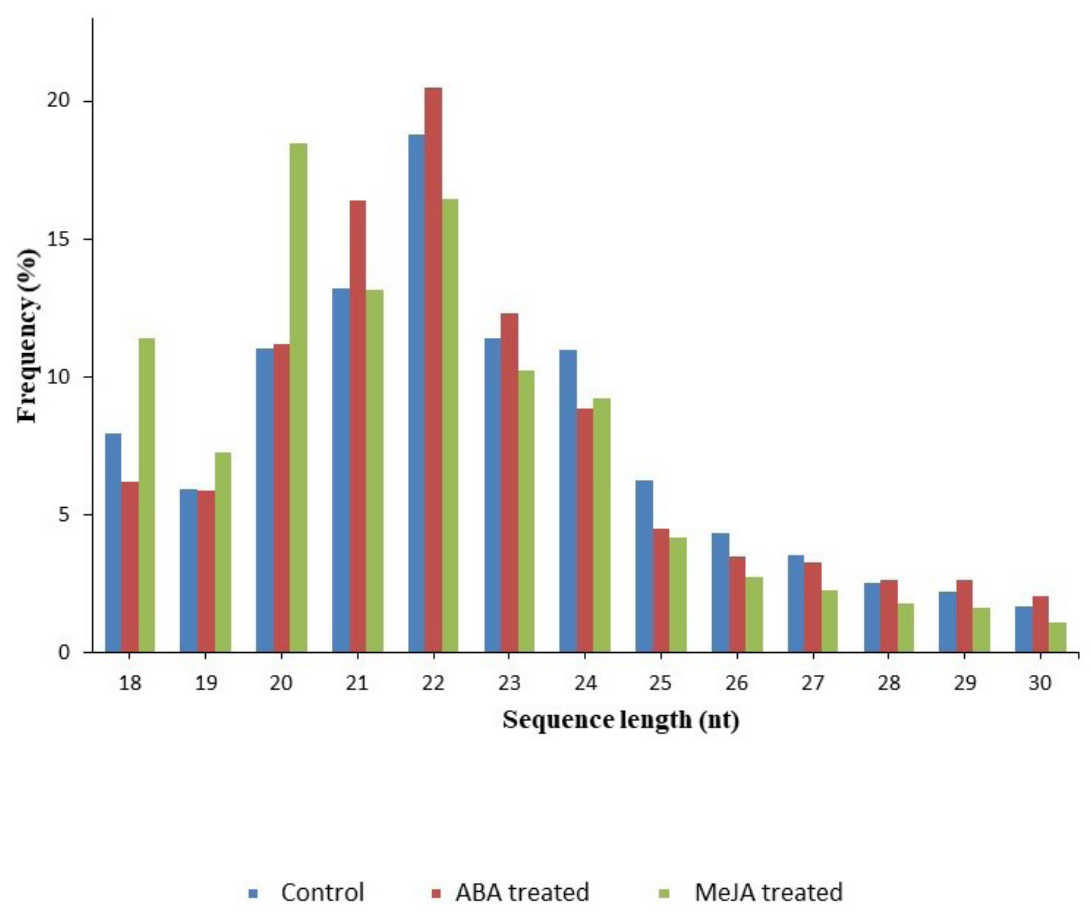

FIGURE 1. Length distribution of small RNA in each library. Distribution of small RNA sequence derived from $\mathrm{K}, \mathrm{ABA}$ and MeJA treated libraries. Majority of the generated reads were $22(>20 \%), 20(>15 \%)$, and 21 (> $15 \%)$ nucleotides

Analysis of miRNA base compositions revealed that uracil was the dominant first base while cytosine was the most dominant at the 19th base (Figure 2). This finding was similar with previous study in soybean which indicated that these two bases may have crucial role in miRNA biogenesis and/or miRNA-mediated gene regulation (Zhang et al. 2008). In total, 173 conserved miRNAs which belong to 62 families were identified (Table 4). In order to unravel novel miRNA in $P$. minor, the unannotated sequences of $\mathrm{K}, \mathrm{ABA}$ and MeJA libraries were searched against transcriptome for the potential miRNA precursors. After the folding prediction and MFEI calculation, 47 unique sequences of putative novel miRNA were discovered in $P$. minor (Table 5). Based on parameters established by Zhang et al. (2006), a secondary structure must have MFEI at least 0.85 to be recognised as precursor miRNA. Table 5 shows all the miRNA precursors that had been discovered in this study that possessed MFEI of at least 0.85 . In addition, all the structures of miRNA precursors were documented in Table 6. 


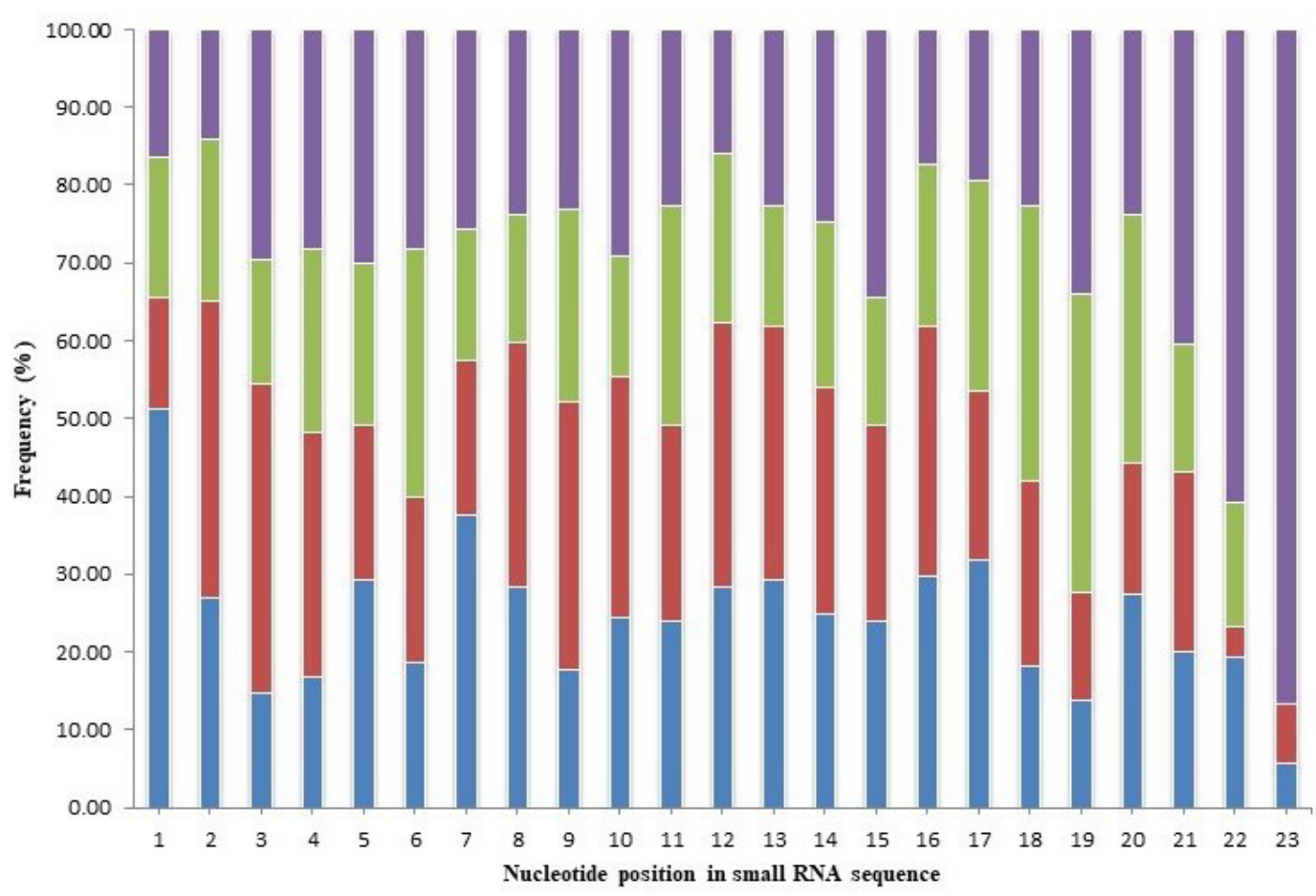

$=\mathrm{A} \quad=\mathrm{C} \quad=\mathrm{G} \quad \| \mathrm{U}$

FIGURE 2. First nucleotide bias in small RNA libraries

TABLE 4. List of conserved miRNAs identified in P. minor

\begin{tabular}{cccccc}
\hline $\begin{array}{c}\text { miRNA } \\
\text { family }\end{array}$ & miRNA & miRnA mature sequce ( 5'-3') & $\begin{array}{c}\text { Sequence } \\
\text { length }\end{array}$ & Conserved miRNA & Plant species \\
\hline 156 & pmi-miR156 & TTGACAGAAGAGAGTGAGCACA & 22 & tae-miR156 & Triticum aestivum \\
& pmi-miR156a & TGACAGAAGAGAGTGAGCACAA & 22 & bna-miR156a & Brassica napus \\
& pmi-miR156b & TGACAGAAGAGAGTGAGCATA & 21 & cca-miR156b & Cynara cardunculus \\
& pmi-miR156c & TTGACAGAAGATAGAGAGCGA & 21 & gma-miR156c & Glycine max \\
& pmi-miR156d-3p & GCTCTCTGTGCTTCTGTCATCA & 22 & stu-miR156d-3p & Solanum tuberosum \\
& pmi-miR156f & TTGACAGAAGAGAGAGAGCATA & 22 & gma-miR156f & Glycine max \\
& pmi-miR156i-3p & TGCTCACTTCTCTTTCTGTCA & 21 & mtr-miR156i-3p & Medicago truncatula \\
& pmi-miR156j & TTGACAGAAGAGGGTGAGCA & 20 & mtr-miR156j & Medicago truncatula \\
& pmi-miR156k & TTGACAGAAGAGAGTGAGCA & 20 & gma-miR156k & Glycine max \\
& pmi-miR1561-3p & GCTCACTTCTCTTTCTGTCAGCA & 23 & osa-miR1561-3p & Oryza sativa \\
& pmi-miR156p & CTGACAGAAGATAGAGAGCA & 20 & mdm-miR156p & Malus domestica \\
& pmi-miR156q & TGACAGAAGAGAGTGAGCACTA & 22 & gma-miR156q & Glycine max \\
& pmi-miR156r & CTGACAGAAGATAGAGAGCATA & 22 & gma-miR156r & Glycine max
\end{tabular}




pmi-miR159
pmi-miR159a
pmi-miR159b-3p
pmi-miR159c
pmi-miR159f
pmi-miR160
pmi-miR156a-3p
pmi-miR156c

162

164

165

166

167

pmi-miR162
pmi-miR162-5p
pmi-miR162a
pmi-miR162b
pmi-miR162b-5p

pmi-miR164a

pmi-miR164b-5p

pmi-miR164e-5p

pmi-miR164g-3p

pmi-miR165a-3p
pmi-miR165b
pmi-miR165c-5p

pmi-miR166

pmi-miR166a-5p

pmi-miR166b

pmi-miR166b-3p

pmi-miR166c

pmi-miR166d

pmi-miR166e

pmi-miR166f-5p

pmi-miR166h-5p

pmi-miR166i

pmi-miR166j-3p

pmi-miR166

pmi-miR1661-3p

pmi-miR166m

pmi-miR166m-5p

pmi-miR166p

pmi-miR166q
TTTGGATCGAAGGGAGCTCTA 21

TTTGGATTGAAGGGAGCTCTATTA 24

TTTGGATTGAAGGGAGCTCTTCA 23

CTTGGATTGAAGGGAGCTCTA 21

CTTGGATTGAAGGGAGCTCCTA

GCGTATGAGGAGCCAAGCATA

TGCCTGGCTCCCTGTATGCCGA

CCTGGCTCCCTGTATGCCATTA

TCGATAAACCTCTGCATCCAA

TGGAGGCAGCGGTTCATCGATCA

TCGATAAACCTCTGCATCCA

TCGATAAGCCTCTGCATCCAGA

GGAGGCAGCGGTTCATCGATCA

pmi-miR167-5p

pmi-miR167a

pmi-miR167b

pmi-miR167c
TGGAGAAGCAGGGCACGTGA

TGGAGAAGCAGGGCACGTGCA

TGGAGAAGCAGGGCACGTGCAA

CACGTGCTCCCCTTCTCCACCA

TCGGACCAGGCTTCATCCCCA

TAGGACCAGGCTTCATCCCCA

GGAATGTTGTCTGGTGCGAGGA

TCGGACCAGGCTTCATTCCCCCA

GGAATGTTGTCTGGCTCGAGGA

TCGGACCAGGCTTCATTCCTA

TCGGACCAGGCTTCATTCCCCA

TCGGACCAGGCTTCATTCCTTA

TCGGACCAGGCTTCATTCCCTA

TCGGACCAGGCTTCATTCCTCA

TGAATGTTGCCTGGCTCGACA

GGAATGTTGGCTGGCTCGAGGTA

TCGGACCAGGCTTCATTCTA

TCGGACCAGGCTTCATTCCCGCA

CCGGACCAGGCTTCATTCCCA

TCGGACCAGGCTTCATCCCTCAA

CGGACCAGGCTTCATTCCCCA

GGAATGTTGGCTGGCTCGAGTCA

TCGGACCAGGCTCCATTCCA

TCGGACCAGGCTTCATTCCTTCA

TGAAGCTGCCAGCATGATCTTTA

TGAAGCTGCCAGCATGATCTCA

TGAAGCTGCCAGCATGATCTAAA

TGAAGCTGCCAGCATGATCTA

\begin{abstract}
atr-miR159
ath-miR159a

aly-miR 159b-3p

sof-miR159c

osa-miR159f
\end{abstract}

csi-miR160

gma-miR160a-3p

mes-miR160c

aau-miR162

csi-miR162-5p

gma-miR162a

osa-miR162b

aly-miR 162b-5p

hci-miR164a

ata-miR164b-5p

bra-mir164e-5p

zma-miR164g-3p

ath-miR165a-3p

ath-miR165b

osa-miR165c-5p

ctr-miR166

aly- miR166a-5p

mtr- miR166b

ata- miR166b-3p

aqc- miR166c

csi- miR166d

cme- miR166e

aly- miR166f-5p

osa- miR166h-5p

cme- miR166i

gma- miR166j-3p

ppt-miR166j/k/1

zma-miR1661-3p

gma- miR166m

zma- miR166m-5p

ptc- miR166p

ptc- miR166q

ahy-miR167-5p

lus-miR167a

cme-miR167b

ata-miR $167 \mathrm{c}$
Amborella trichopoda

Arabidopsis thaliana

Arabidopsis lyrata

Saccharum officinarum

Oryza sativa

Citrus sinensis

Glycine $\max$

Manihot esculenta

Acacia auriculiformis

Citrus sinensis

Glycine max

Oryza sativa

Arabidopsis lyrata

Helianthus ciliaris

Aegilops tauschii

Brassica rapa

Zea mays

Arabidopsis thaliana

Arabidopsis thaliana

Oryza sativa

\section{Citrus trifoliate}

Arabidopsis lyrata

Medicago truncatula

Aegilops tauschii

Aquilegia caerulea

Citrus sinensis

Cucumis melo

Arabidopsis lyrata

Oryza sativa

Cucumis melo

Glycine max

Phsycomitrella patens

Zea mays

Glycine max

Zea mays

Populus trichocarpa

Populus trichocarpa

Arachis hypogaea

Linum usitatissimum

Cucumis melo

Aegilops tauschii 


pmi-miR167c-5p
pmi-miR167d
pmi-miR167f-5p
pmi-miR167h

168

169

171

pmi-miR168
pmi-miR168a
pmi-miR168a-3p
pmi-miR168b-3p
pmi-miR168c-5p

pmi-miR168c-5p

$$
\begin{aligned}
& \text { pmi-miR169f } \\
& \text { pmi-miR169h } \\
& \text { pmi-miR169i }
\end{aligned}
$$

71

$$
\begin{gathered}
\text { pmi-miR171 } \\
\text { pmi-miR171a } \\
\text { pmi-miR171c-3p } \\
\text { pmi-miR171c-5p } \\
\text { pmi-miR171d }
\end{gathered}
$$

$$
\begin{aligned}
& \text { pmi-miR172a } \\
& \text { pmi-miR172a-3p } \\
& \text { pmi-miR172b } \\
& \text { pmi-miR172c } \\
& \text { pmi-miR172d } \\
& \text { pmi-miR172d-5p } \\
& \text { pmi-miR172f } \\
& \text { pmi-miR172h } \\
& \text { pmi-miR172i } \\
& \text { pmi-miR172m }
\end{aligned}
$$

pmi-miR319c-3p pmi-miR319e pmi-miR319i pmi-miR390a-5p pmi-miR390b pmi-miR390d

pmi-miR391-5p
TAAGCTGCCAGCATGATCTTA TAAGCTGCCAGCATGATCTGGA

TAAGCTGCCAGCATGATCTGCTA TAAAGCTGCCAGCATGATCTTA

TCGCTTGGTGCAGGTCGGGAA

TCGCTTGGTGCAGGTCGGGAACA

$$
\text { CCCGCCTTGCATCAACTGAATCA }
$$

CCCGCCTTGCATCAACTGAATA

TCGCTTGGTGCAGGTCGGGATA

\section{TAGCCAGGGATGACTTGCCGGA AGGCAGTCTCCTTGACTATTA \\ TAGCCAAGGACGACTTGCCTGA}

TGATTGAGCCGCGCCAATATCA TGAGCCGCGCCAATATCA

TTGAGCCGTGCCAATATCA GGATATTGGTGCGGTTCAATCA TTGAGCCGTGCCAATATCACGA

AGAATCTTGATGATGCTGCAGTA

AGAATCTTGATGATGCTGCAA

AGAATCTTGATGATGCTACACA GGAGCATCATCAAGATTCACA AGAATCTTGATGATGCTGCAGCA GGAGCATCATCAAGATTCACATA AGAATCTTGATGATGCTGCATCA GCAGCAGCATCAAGATTCACA AGAATCTTGATGATGCTGCATTA AGAATCTTGATGATGCTGCAGCA

TTGGACTGAAGGGAGCTCCCTA CTTGGACTGAAGGGAGCTCCA TTGGACTGAAGGGAGCTCCCTTA CTTGGACTGAAGGGAGCTCCCA TTGGACTGAAGGGAGCTCCCA CTTGGACTGAAGGGAGCTCCCAA 23 TTGGGCTGAAGGGAGCTCCCA 21 AAGCTCAGGAGGGATAGCGCCA 22 AAGCTCAGGAGGGATAGCGCCCA 23 AAGCTCAGGAGGGATAGCACCA 22

CTTCGCAGGAGCGATGGCGCCA

$$
\begin{array}{cc}
\begin{array}{c}
\text { aly-miR167c-5p } \\
\text { ath-miR167d }
\end{array} & \text { Arabidopsis lyrata } \\
\text { ata-miR167f-5p } & \text { Arabidopsis thaliana } \\
\text { mdm-miR167h } & \text { Malus domestica }
\end{array}
$$$$
\text { atr-miR168 }
$$$$
\text { cca-miR168a }
$$$$
\text { aly-miR168a-3p }
$$$$
\text { sly-miR168b-3p }
$$$$
\text { bra-miR168c-5p }
$$

mes-miR169f

aly-miR169h-3p

aly-miR169i

$$
\begin{gathered}
\text { ccl-miR171 } \\
\text { csi-miR171a } \\
\text { ata-miR171c-3p } \\
\text { osa-miR171c-5p } \\
\text { bna-miR171d } \\
\text { lja-miR172a } \\
\text { csi-miR172a-3p } \\
\text { vvi-miR172b } \\
\text { aly-miR172c } \\
\text { gma-miR172d } \\
\text { stu-miR172d-5p } \\
\text { nta-miR172f } \\
\text { gma-miR172h-5p } \\
\text { nta-miR172i } \\
\text { mdm-miR172m }
\end{gathered}
$$

aqc-miR319

ppt-miR319a

mdm-miR319b

ppt-miR319c

mtr-miR319c-3p

ppt-miR319e

ptc-miR319i

aly-miR390a-5p/b

ppt-miR390b

gma-miR390d

ath-miR391-5p

$$
\begin{gathered}
\text { ghr-miR393 } \\
\text { ath-miR393a-5p } \\
\text { gma-miR393c-3p }
\end{gathered}
$$

$$
\begin{gathered}
\text { Amborella trichopoda } \\
\text { Cynara cardunculus } \\
\text { Arabidopsis lyrata } \\
\text { Solanum lycopersicum } \\
\text { brassica rapa } \\
\text { Monihot esculenta } \\
\text { Arabidopsis lyrata } \\
\text { Arabidopsis lyrata }
\end{gathered}
$$

\section{Monihot esculenta \\ Arabidopsis lyrata \\ Arabidopsis lyrata}

Citrus clementina

Citrus sinensis

Aegilops tauschii

Oryza sativa

Brassica napus

Lotus japonicas

Citrus sinensis

Vitis vinifera

Arabidopsis lyrata

Glycine max

Solanum tuberosum

Nicotiana tabacum

Glycine max

Nicotiana tabacum

Malus domestica

Aquilegia caerulea

Phsycomitrella patens

Malus domestica

Phsycomitrella patens

Medicago truncatula

Phsycomitrella patens

Populus trichocarpa

Arabidopsis lyrata

Phsycomitrella patens

Glycine max

Arabidopsis thaliana

Gossypium hirsutum

Arabidopsis thaliana

Glycine max 


$$
\begin{gathered}
\text { pmi-miR394 } \\
\text { pmi-miR394a } \\
\text { pmi-miR394b-5p }
\end{gathered}
$$

pmi-miR395

pmi-miR395a

pmi-miR395b

pmi-miR395d

$$
\begin{gathered}
\text { pmi-miR396 } \\
\text { pmi-miR396a } \\
\text { pmi-miR396b-3p } \\
\text { pmi-miR396b-5p } \\
\text { pmi-miR396c } \\
\text { pmi-miR396e-3p }
\end{gathered}
$$

pmi-miR397

pmi-miR397a

pmi-miR397b

$$
\begin{gathered}
\text { pmi-miR398 } \\
\text { pmi-miR398 } \\
\text { pmi-miR398a } \\
\text { pmi-miR398b } \\
\text { pmi-miR398c-5p } \\
\text { pmi-miR398f }
\end{gathered}
$$

$$
\begin{gathered}
\text { pmi-miR399 } \\
\text { pmi-miR399b } \\
\text { pmi-miR399f } \\
\text { pmi-miR399h-5p } \\
\text { pmi-miR399j } \\
\text { pmi-miR408 } \\
\text { pmi-miR408-3p } \\
\text { pmi-miR530b } \\
\text { pmi-miR535 } \\
\text { pmi-miR535a } \\
\text { pmi-miR535b } \\
\text { pmi-miR535d } \\
\text { pmi-miR828a } \\
\text { pmi-miR833a-5p } \\
\text { pmi-miR845a } \\
\text { pmi-miR845c } \\
\text { pmi-miR845d/e } \\
\text { ath-miR858b } \\
\text { ppt-miR894 } \\
\text { pmi-miR1127b-3p } \\
\text { pmi-miR1128 } \\
\text { pmi-miR1436 } \\
\text { pmi-miR1439 }
\end{gathered}
$$

$$
\begin{aligned}
& \text { TTGGCATTCTGTCCATCTCCA } \\
& \text { TTGGCATTCTGTCCATCTCCTTA }
\end{aligned}
$$$$
\text { TTGGCATTCTGTCCACCTCCTA }
$$$$
\text { CTGAAGCGTTTGGGGGAACGA }
$$$$
\text { TGAAGTGTTTGGGGGAACTCCA }
$$$$
\text { TGAAGTGTTTAGGGGAACTCGA }
$$$$
\text { TGAAGTGTTTGGGGGAACTCTA }
$$

$$
\begin{gathered}
\text { TTCCACAGCTTTCTTGAACTGCA } \\
\text { GTTCAATAAAGCTGTGGGA } \\
\text { GTTCAATAAAGCTGTTGGAA } \\
\text { TTCCACAGCTTTCTTGAACTTTA } \\
\text { TTCAAGAAAGCTGTGGGAAAA } \\
\text { TTCAATAAAGCTGTGGGAAA }
\end{gathered}
$$

TCATTGAGTGCAGCGTTGACGA

TCATTGAGTGCAGCGTTGATGTA

TTATTGAGTGCAGCGTTGATGA

TGTGTTCCCAGGTCGCCCCTGA GGAGCGACCTGAGATCACATGA TGTGTTCTCAGGTCGCCCCTGCA TGTGTTCTCAGGTCGCCCCTG GGAGCGACCTGAAACCACATGA GGTGTTCTCAGGTCGCCCCTAA

TGCCAAAGGAGAGTTGCCCTA TGCCAAAGGAGAGTTGCCCTGA TGCCAAAGGAGATTTGCCCGGA GGGCAAGATCTCTATTGGCAGGA TGCCAAAGGAGAGTTGCCCTAA TGCACTGCCTCTTCCCTGGCTA ATGCACTGCCTCTTCCCTGGCA TGCATTTGCACCTACACCTTA TGACAATGAGAGAGAGCACA TGACAATGAGAGAGAGCACGT TGACAATGAGAGAGAGCACGGA TGACGATGAGAGAGAGCACGA TCTTGCTCAAATGAGTATTCCA GTTTGTTGTGCTCGGTCTA CGGCTCTGATACCAATTGTTA AGGCTCTGATACCAATTGAAGCA TGGCTCTGATACCAATTGACGCA TTCGTTGTCTGTTCGACCTTGA CGTTTCACGTCGGGTTCACCAA ACATGTATTTTTGGACGGAGGGA CTACTACCTCCGTCTCAAAAA ATTATGGAACGGAGGGAGTA TTTTGGAACGGAGAGAGTA

$$
\begin{gathered}
\text { cca-miR394 } \\
\text { vvi-miR319a } \\
\text { ptc-miR394b-5p } \\
\text { ppt-395 } \\
\text { sly-miR395a } \\
\text { tea-miR395b } \\
\text { rco-miR395d }
\end{gathered}
$$

$$
\begin{gathered}
\text { aau-miR396 } \\
\text { vvi-miR396a } \\
\text { zma-miR396b-3p } \\
\text { ath-miR396b-5p } \\
\text { cca-miR396c } \\
\text { ata-miR396e-3p }
\end{gathered}
$$

Cynara cardunculus Vitis vinifera

Populus trichocarpa Physcomitrella patens

Solanum lycopersicum

Triticum aestivum

Ricinus communis

$$
\begin{aligned}
& \text { aqc-miR399 } \\
& \text { ath-miR399b } \\
& \text { ath-miR399f } \\
& \text { aly-miR399h-5p } \\
& \text { osa-miR399j } \\
& \text { cca-miR408 } \\
& \text { ath-miR408-3p } \\
& \text { cme-miR530b } \\
& \text { csi-miR535 } \\
& \text { mes-miR535a } \\
& \text { mes-miR535b } \\
& \text { mdm-miR535d } \\
& \text { vvi-miR828a } \\
& \text { ath-miR833a-5p } \\
& \text { ath-miR845a } \\
& \text { vvi-miR845c } \\
& \text { vvi-miR845d/e } \\
& \text { ath-miR858b } \\
& \text { ppt-miR894 } \\
& \text { tae-miR1127b-3p } \\
& \text { ssp-miR1128 } \\
& \text { hvu-miR1436 } \\
& \text { osa-miR1439 }
\end{aligned}
$$

Acacia auriculiformis

Vitis vinifera

Zea mays

Arabidopsis thaliana

Cynara cardunculus

Aegilops tauschii

Picea abies

Brassica napus

Oryza sativa

\section{Amborella trichopoda \\ Hevea brasiliensis \\ Cucumis melo \\ Oryza sativa \\ Populus trichocarpa \\ Linum usitatissimum}

Arabidopsis thaliana

Arabidopsis thaliana

Arabidopsis thaliana

Arabidopsis lyrata

Oryza sativa

Cynara cardunculus

Arabidopsis thaliana

Cucumis melo

Citrus sinensis

Monihot esculenta

Monihot esculenta

Molus domesticus

Vitis vinifera

Arabidopsis thaliana

Arabidopsis thaliana

Vitis vinifera

Vitis vinifera

Arabidopsis thaliana

Phsycomitrella patens

Triticum aestivum

Saccharum sp.

Hordeum vulgare

Oryza sativa 


\begin{tabular}{|c|c|c|c|c|c|}
\hline 1511 & pmi-miR1511-3p & ACCTGGCTCTGATACCATA & 19 & ppe-miR1511-3p & Prunus persica \\
\hline \multirow[t]{2}{*}{1863} & pmi-miR1863 & AAGCTCTGATACCATGTTAGATTTA & 25 & cme-miR1863 & Cucumis melo \\
\hline & pmi-miR1863a & AAGCTCTGATACCATGTTAGATTA & 24 & osa-miR1863a & Oryza sativa \\
\hline 1874 & pmi-miR1874-5p & AGGGCTACTATAACATCCATA & 21 & osa-miR1874-5p & Oryza sativa \\
\hline 2673 & pmi-miR2673a/b & СТСТТТСТСТТССТСТТССАА & 21 & mtr-miR2673a/b & Medicago truncatula \\
\hline 2916 & pmi-miR2916 & TGGGGGCTCGAAGACGATCAGATA & 24 & peu-miR2916 & Populus euphratica \\
\hline 3627 & pmi-miR3627a & CTTCGCAGGAGCGATGGCACTA & 22 & mdm-miR3627a & Malus domestica \\
\hline 3630 & pmi-miR3630-3p & TGGGAATCTCTCTGATGCACA & 21 & vvi-miR3630-3p & Vitis vinifera \\
\hline 4995 & pmi-miR4995 & TAGGCAGTGGCTTGGTTAAGGGAA & 24 & gma-miR4995 & Glycine max \\
\hline 5049 & pmi-miR5049c & AGACAATTATTGTGGGACGGAGGAA & 25 & hvu-miR5049c & Hordeum vulgare \\
\hline 5054 & pmi-miR5054 & TCCCCACGGACGGCGCCAA & 19 & bdi-miR5054 & Brachypodium distachyon \\
\hline 5056 & pmi-miR5056 & GAGGAAGAACCGGTAATAGACA & 22 & bdi-miR5056 & Brachypodium distachyon \\
\hline 5077 & pmi-miR5077 & TTCGCGTCGGGTTCACCAA & 19 & osa-miR5077 & Oryza sativa \\
\hline 5083 & pmi-miR5083 & CAGACTACAATTATCTGATCAA & 22 & osa-miR5083 & Oryza sativa \\
\hline 5139 & pmi-miR5139 & AAAACTTGGCTCTGATACCA & 20 & rgl-miR5139 & Rehmannia glutinosa \\
\hline \multirow[t]{2}{*}{5174} & $\begin{array}{c}\text { pmi-miR5174d- } \\
3 \mathrm{p}\end{array}$ & CAATCTTTTTGGATCGGAGAGAGTA & 25 & bdi-miR5174d-3p & Brachypodium distachyon \\
\hline & pmi-miR5174e-5p & ACTCCCTCTGTTCCATAA & 18 & bdi-miR5174e-5p.2 & Brachypodium distachyon \\
\hline 5181 & pmi-miR5181-3p & ACACTTATTTTGGAACAGAGGGA & 23 & ata-miR5181-3p & Aegilops tauschii \\
\hline 5368 & pmi-miR5368 & GGACAGTCTCAGGTAGACA & 19 & gma-miR5368 & Glycine $\max$ \\
\hline 5532 & pmi-miR5532 & ATGGAATATATGACAAGGGTGTA & 23 & osa-miR5532 & Oryza sativa \\
\hline 5538 & pmi-miR5538 & CTACTGAACTCAATCACTTGCTA & 23 & osa-miR5538 & Oryza sativa \\
\hline 5658 & pmi-miR5658 & TGATGATGAAGATGATGAA & 19 & ath-miR5658 & Arabidopsis thaliana \\
\hline 6173 & pmi-miR6173 & GAGCCGTAAACGATGGATA & 22 & hbr-miR6173 & Hevea brasiliensis \\
\hline 6300 & pmi-miR6300 & GTCGTTGTAGTATAGTGGA & 18 & gma-miR6300 & Glycine max \\
\hline 6478 & pmi-miR6478 & CCGACCTTAGCTCAGTTGGTGA & 22 & ptc-miR6478 & Populus trichocarpa \\
\hline 6485 & pmi-miR6485 & AGGATGTAGAAGATCATAACA & 21 & hbr-miR6485 & Hevea brasiliensis \\
\hline 7729 & $\begin{array}{l}\text { pmii-miR7729a/ } \\
\text { b-3p }\end{array}$ & CAATGGTGGTGGTTGGGAGGA & 21 & bdi-miR7729a/b-3p & Brachypodium distachyon \\
\hline 7767 & pmi-miR7767-5p & CCCCAAGATGAGTGCTCTCCCA & 22 & bdi-miR7767-5p & Brachypodium distachyon \\
\hline 8175 & pmi-miR8175 & CGATCCCCGGCAACGGCGCCAA & 22 & ath-miR8175 & Arabidopsis thaliana \\
\hline 9670 & pmi- miR9670-3p & AGGTGGAAAACCTGAAGAAGA & 21 & tae-miR9670-3p & Triticum aestivum \\
\hline
\end{tabular}

TABLE 5. List of putative novel miRNAs that had been discovered in P. minor

\begin{tabular}{|c|c|c|c|c|c|c|c|c|c|}
\hline Novel miRNA & Mature sequences & LM & LP & $\begin{array}{l}\text { Side } \\
\text { Arm }\end{array}$ & $\Delta \mathrm{G}$ & $\begin{array}{c}A+U \\
(\%)\end{array}$ & $\begin{array}{c}\mathrm{G}+\mathrm{C} \\
(\%)\end{array}$ & AMFE & MFEI \\
\hline pmi-miRNew-01 & GGGGAAACTGTTGGGCCA & 18 & 73 & 5 , & -28.6 & 60.27 & 39.73 & 39.18 & 1.01 \\
\hline pmi-miRNew-02 & TAAACGAGCCGAGTATGAGCA & 21 & 93 & 3 ' & -29.5 & 62.37 & 37.63 & 31.72 & 1.19 \\
\hline pmi-miRNew-03 & TGTCAGAACTAAGTGTGGGGGA & 22 & 172 & 3 ' & -43.4 & 60.47 & 39.53 & 25.23 & 1.57 \\
\hline pmi-miRNew-04 & TTGTATCTAGGGCTCATAAGATA & 23 & 133 & $3^{\prime}$ & -46.5 & 57.89 & 42.11 & 34.96 & 1.20 \\
\hline pmi-miRNew-05 & GTGCTCTCTCTCATTGTCATA & 20 & 103 & 3 ' & -57.2 & 56.31 & 43.69 & 55.53 & 0.99 \\
\hline pmi-miRNew-06 & TGGTAGATGTGCTTGTCAAGCA & 22 & 93 & 5 ' & -35.7 & 48.39 & 51.61 & 38.39 & 1.34 \\
\hline pmi-miRNew-07 & CGTCTCGTCGCCCTTAGATCGA & 22 & 103 & 5 ' & -64.3 & 40.78 & 59.22 & 62.43 & 0.95 \\
\hline pmi-miRNew-08 & GGAGCGACCTTAGACCACATGA & 22 & 143 & 5 ' & -59.0 & 47.55 & 52.45 & 41.26 & 1.27 \\
\hline pmi-miRNew-09 & CCTTTGTCGCATTTGGGGAAA & 21 & 143 & 3 ' & -76.1 & 58.04 & 41.96 & 53.22 & 0.99 \\
\hline pmi-miRNew-10 & CATTTCTGGTGGTAGCTCATA & 21 & 73 & 5 , & -19.9 & 63.01 & 36.99 & 27.26 & 1.36 \\
\hline
\end{tabular}




\begin{tabular}{|c|c|c|c|c|c|c|c|c|c|}
\hline pmi-miRNew-11 & CGGGGAAGAGGCTGAGCAAGGA & 22 & 103 & 5 & -57.5 & 50.49 & 49.51 & 55.83 & 0.89 \\
\hline pmi-miRNew-12 & TGAATTGTGTGTGAATGA & 18 & 83 & $3^{\prime}$ & -18.6 & 73.49 & 26.51 & 12.77 & 2.08 \\
\hline pmi-miRNew-13 & TGTATTTTTGGACGGAGGTAGTA & 23 & 82 & $3^{\prime}$ & -18.8 & 67.07 & 32.93 & 16.83 & 1.96 \\
\hline pmi-miRNew-14 & GTGCTCTCTCTCATTGTCAA & 20 & 123 & 3 & -48.7 & 56.91 & 43.09 & 39.59 & 1.09 \\
\hline pmi-miRNew-15 & GTGGTGGTATTGTGGACAGCA & 21 & 123 & 5 & -35.3 & 50.41 & 49.59 & 28.70 & 1.73 \\
\hline pmi-miRNew-16 & GGAATTATGGCTGTATCGCATA & 21 & 123 & 5 & -22.2 & 67.48 & 32.52 & 18.05 & 1.80 \\
\hline pmi-miRNew-17 & TTCTGATTTGTGATGTAATCCA & 22 & 93 & 3 & -59.7 & 59.14 & 40.86 & 64.19 & 0.85 \\
\hline pmi-miRNew-18 & GCTGAGATTGTAAAGGCTTTTTA & 23 & 103 & 3 & -29.4 & 67.96 & 32.04 & 28.54 & 1.12 \\
\hline pmi-miRNew-19 & CTGTTGGGCTTGCTCTTA & 18 & 82 & 5 & -27.0 & 50.00 & 50.00 & 32.93 & 1.52 \\
\hline pmi-miRNew-20 & TCCСАCТСТCAACACCAA & 18 & 83 & 5 & -28.6 & 44.58 & 55.42 & 34.46 & 1.61 \\
\hline pmi-miRNew-21 & AACGGTGAAACGAATGAATATTG & 23 & 114 & 5 & -34.7 & 72.81 & 27.19 & 30.44 & 0.89 \\
\hline pmi-miRNew-22 & AAGAAGATCAACGGATGAGATTA & 23 & 83 & 5 & -20.4 & 61.45 & 38.55 & 24.58 & 1.57 \\
\hline pmi-miRNew-23 & TGATTGAAATGGTTCTCGACGA & 22 & 63 & 3 & -23.2 & 58.73 & 41.27 & 36.83 & 1.12 \\
\hline pmi-miRNew-24 & ATGGACAGCACTGTATTGGCA & 21 & 83 & 3 & -21.9 & 54.22 & 45.78 & 26.39 & 1.74 \\
\hline pmi-miRNew-25 & TTGCAGAGATTGCCGGTAACA & 21 & 63 & 5 & -18.3 & 55.56 & 44.44 & 24.29 & 1.83 \\
\hline pmi-miRNew-26 & CGAGGCAAGAACTTTGGAGCA & 21 & 103 & 3 & -43.1 & 53.40 & 46.60 & 41.84 & 1.11 \\
\hline pmi-miRNew-27 & CGTGTTATCGTGTCGGATA & 19 & 63 & $3^{\prime}$ & -33.6 & 50.79 & 49.21 & 53.33 & 0.92 \\
\hline pmi-miRNew-28 & GACAGGACCTTTGAAGTAGCA & 21 & 93 & 3 & -24.1 & 49.46 & 50.54 & 25.91 & 1.95 \\
\hline pmi-miRNew-29 & TCAAACACGGGAGTACAACTA & 21 & 123 & 3 & -49.5 & 66.67 & 33.33 & 40.24 & 0.85 \\
\hline pmi-miRNew-30 & TGGGATTTGAGCCACAGATAA & 21 & 113 & 5 & -31.6 & 51.33 & 48.67 & 27.96 & 1.74 \\
\hline pmi-miRNew-31 & CCGGAAGACCTAGAGCTA & 18 & 83 & 5 & -24.7 & 57.83 & 42.17 & 29.76 & 1.42 \\
\hline pmi-miRNew-32 & GATTAATCCGGCATGAGCTA & 20 & 83 & 5 & -29.2 & 50.60 & 49.40 & 35.18 & 1.40 \\
\hline pmi-miRNew-33 & CAGAGGTTAATCGTACTCTGGCA & 23 & 83 & 5 & -20.0 & 60.24 & 39.76 & 24.10 & 1.65 \\
\hline pmi-miRNew-34 & TGGCTCAATGCATGCAACTCA & 21 & 103 & 5 & -50.0 & 54.37 & 45.63 & 48.54 & 0.94 \\
\hline pmi-miRNew-35 & CTGTGACTCAAGAGGGGCA & 19 & 143 & 3 & -70.9 & 60.84 & 39.16 & 49.58 & 0.99 \\
\hline pmi-miRNew-36 & AGGTCACAAATGGACGGTTGA & 21 & 113 & 5 & -60.2 & 49.56 & 50.44 & 53.27 & 0.95 \\
\hline pmi-miRNew-37 & GTCTGTTTATTACATTTTGAA & 21 & 93 & 5 & -21.1 & 68.82 & 31.18 & 22.69 & 1.37 \\
\hline pmi-miRNew-38 & CCAAATCTGAGTTATCTGTCA & 21 & 173 & $3^{\prime}$ & -53.6 & 51.45 & 48.55 & 30.98 & 1.57 \\
\hline pmi-miRNew-39 & TTCTCGTAGGATAATTGTACTA & 22 & 133 & $3^{\prime}$ & -55.1 & 59.40 & 40.60 & 41.43 & 0.98 \\
\hline pmi-miRNew-40 & AGAGATGTTGGCTAAGCAAGA & 21 & 133 & 3 & -56.1 & 60.90 & 39.10 & 42.18 & 0.93 \\
\hline pmi-miRNew-41 & CGATCTGTATGAGAATCTTGA & 22 & 123 & 3 & -59.8 & 59.35 & 40.65 & 48.62 & 0.85 \\
\hline pmi-miRNew-42 & AATGTGCAAATTTGAGCA & 18 & 63 & 3 & -24.6 & 55.56 & 44.44 & 39.05 & 1.14 \\
\hline pmi-miRNew-43 & CTCGAAGAGGAACACAAGATA & 21 & 153 & 3 & -28.0 & 61.44 & 38.56 & 18.30 & 2.11 \\
\hline pmi-miRNew-44 & AGAGATGTGAATGAGACCA & 19 & 123 & 3 & -29.6 & 57.72 & 42.28 & 24.07 & 1.76 \\
\hline pmi-miRNew-45 & TTTTTACTGTTGTCAACTA & 19 & 72 & 5 & -18.2 & 62.50 & 37.50 & 22.50 & 1.67 \\
\hline pmi-miRNew-46 & ACAGAGACGGTCGGGGGTA & 19 & 83 & 3 & -36.7 & 55.42 & 44.58 & 44.22 & 1.01 \\
\hline pmi-miRNew-47 & AGCTAATTGGTTGTTCAAACA & 21 & 103 & $3^{\prime}$ & -37.0 & 58.25 & 41.75 & 35.92 & 1.16 \\
\hline
\end{tabular}

$\mathrm{LM}=$ Length of mature sequence, $\mathrm{LP}=$ Length of precursor sequence, $\Delta \mathrm{G}=$ Free energy, $\mathrm{AMFE}=$ Adjusted minimum folding energy, $\mathrm{MFEI}=\mathrm{Minimum}$ folding energy index 
pmi-miRNew-01

pmi-miRNew-02

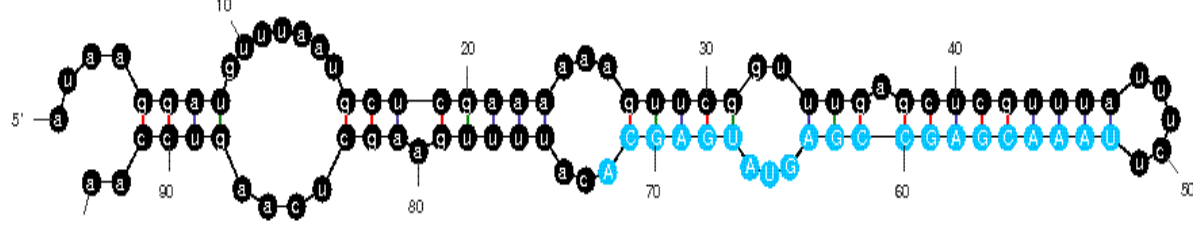

pmi-miRNew-03

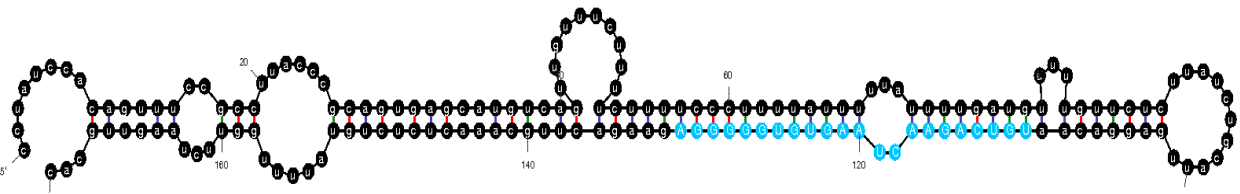

pmi-miRNew-04

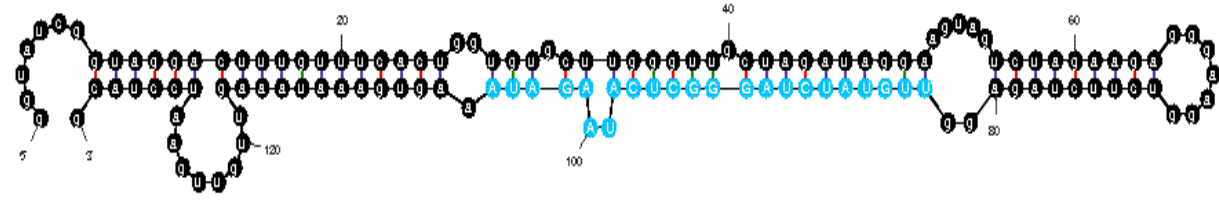

pmi-miRNew-05

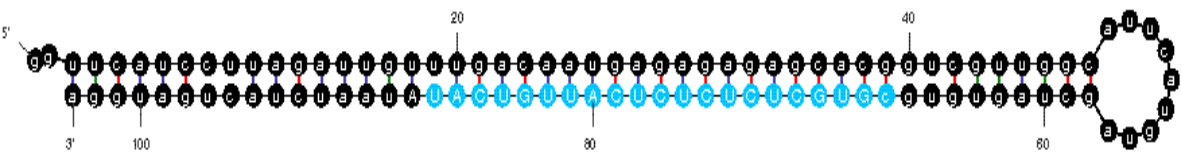

pmi-miRNew-06

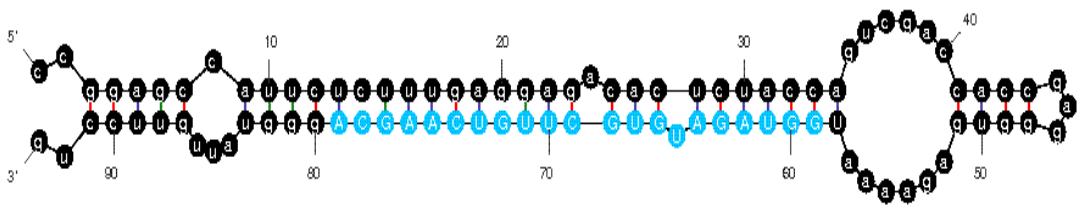


pmi-miRNew-06*
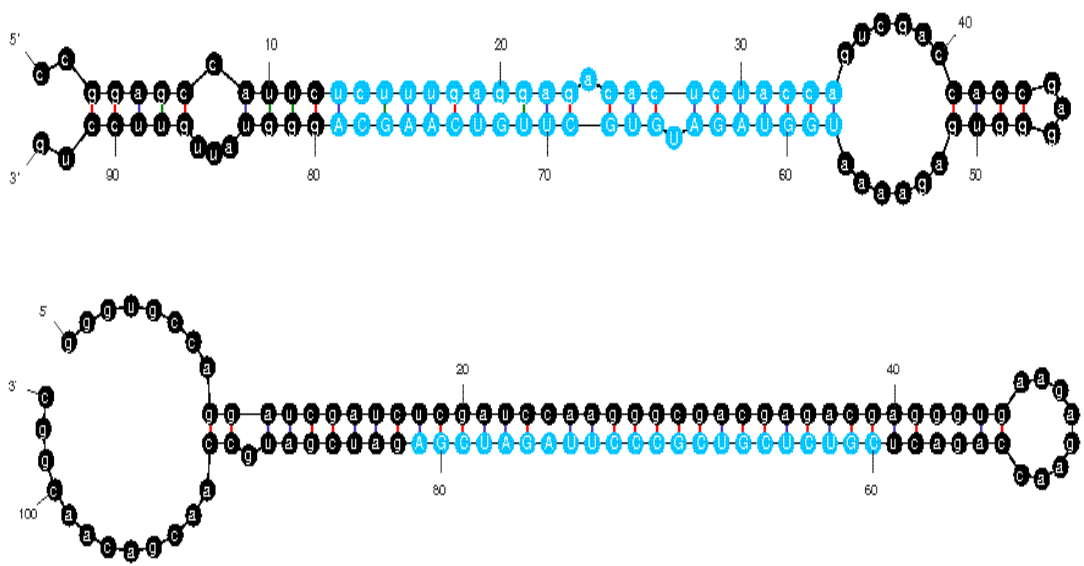

pmi-miRNew-07*

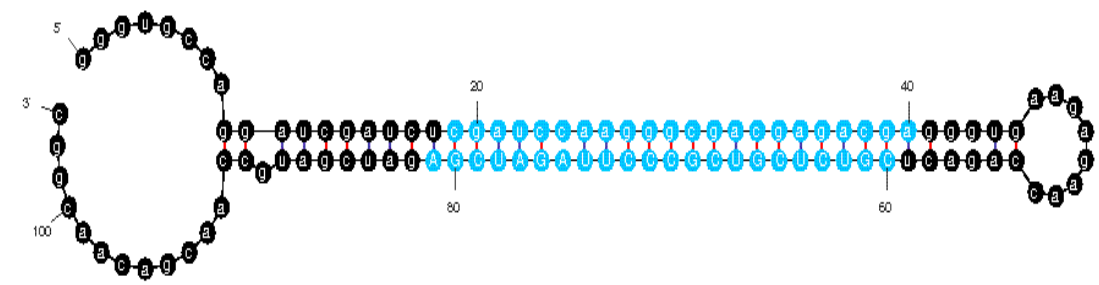

pmi-miRNew-08

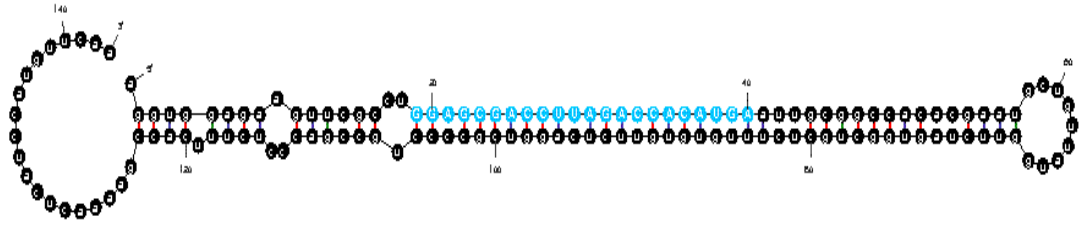

pmi-miRNew-09

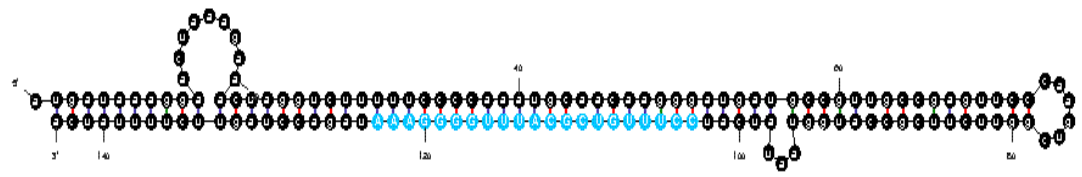

pmi-miRNew-10

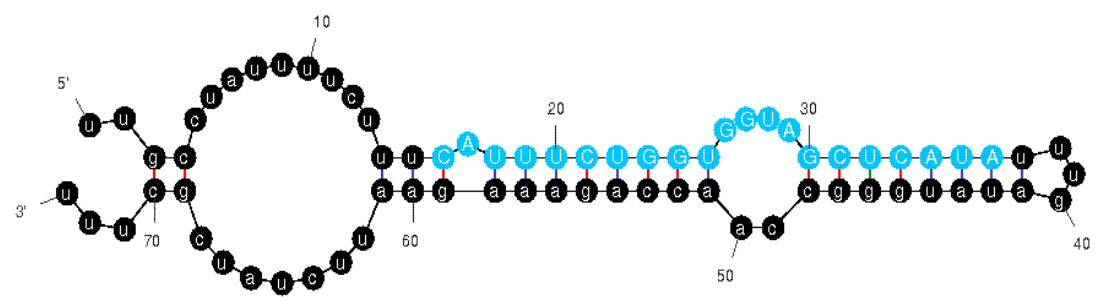

pmi-miRNew-11

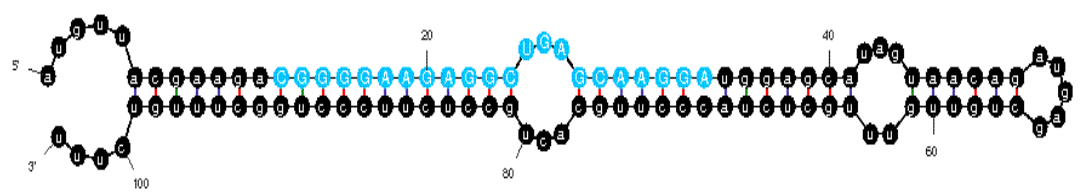


pmi-miRNew-12

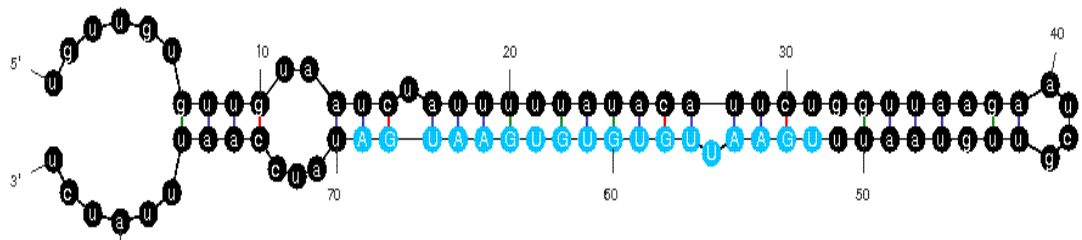

pmi-miRNew-13

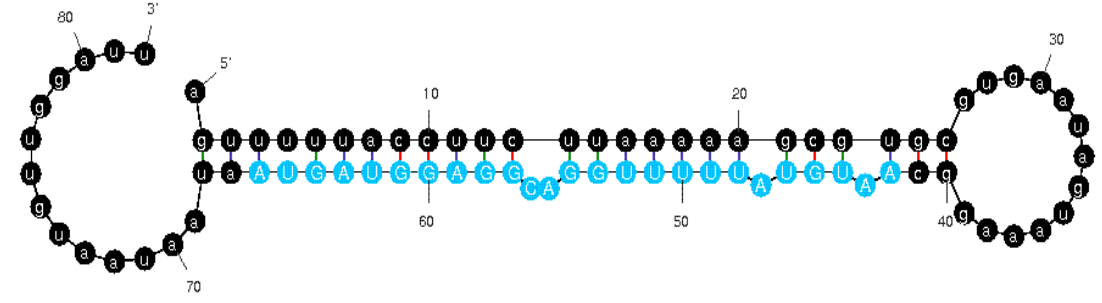

pmi-miRNew-14

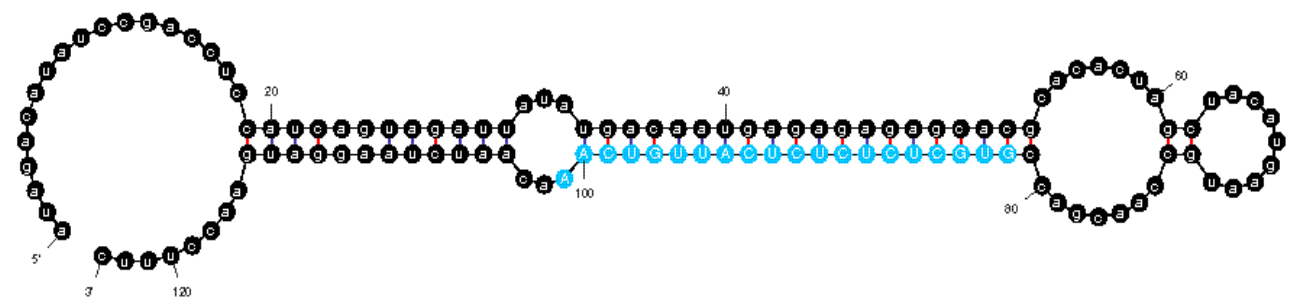

pmi-miRNew-15

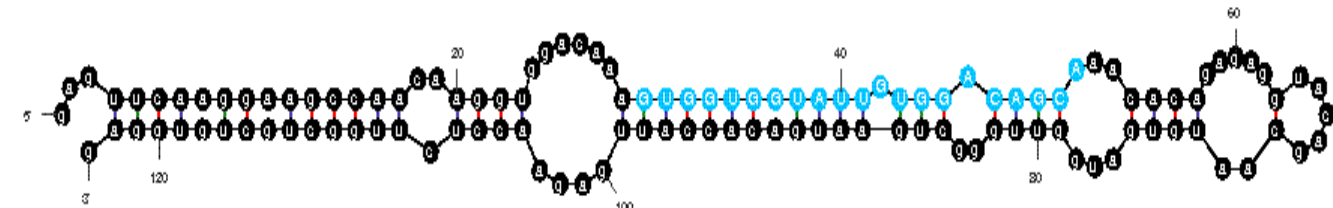

pmi-miRNew-15*

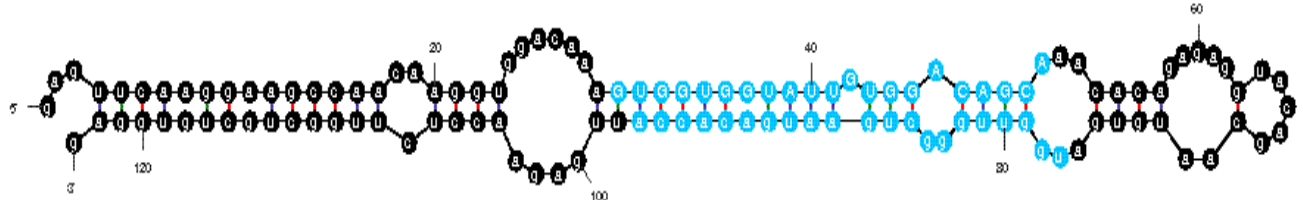

pmi-miRNew-16

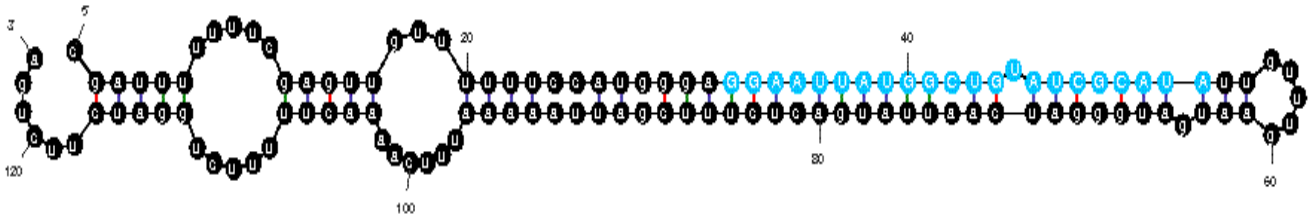

pmi-miRNew-17

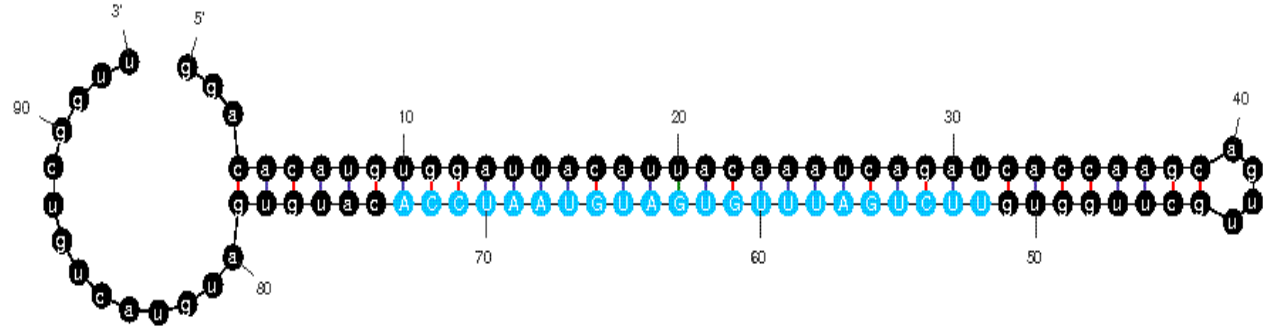


pmi-miRNew-18

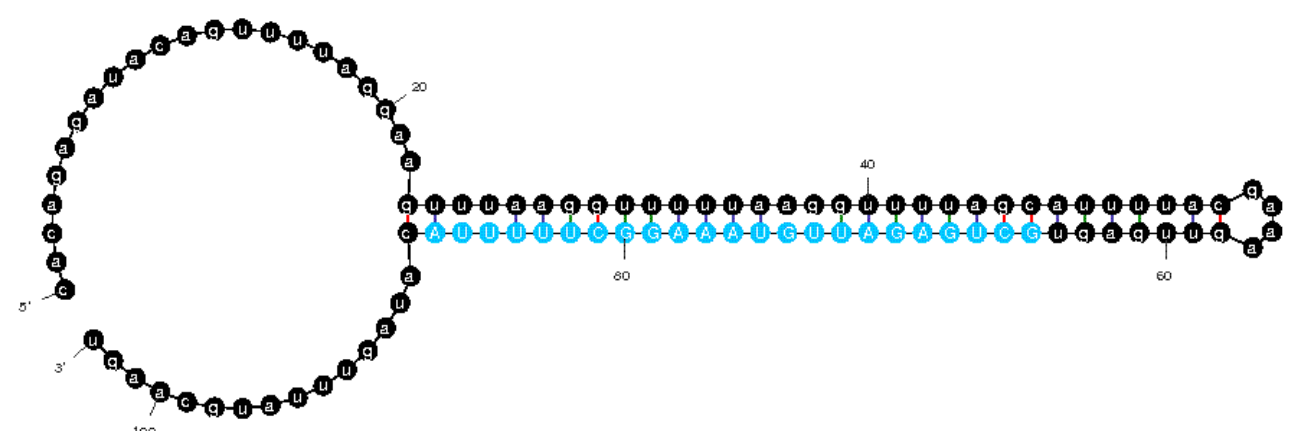

pmi-miRNew-19

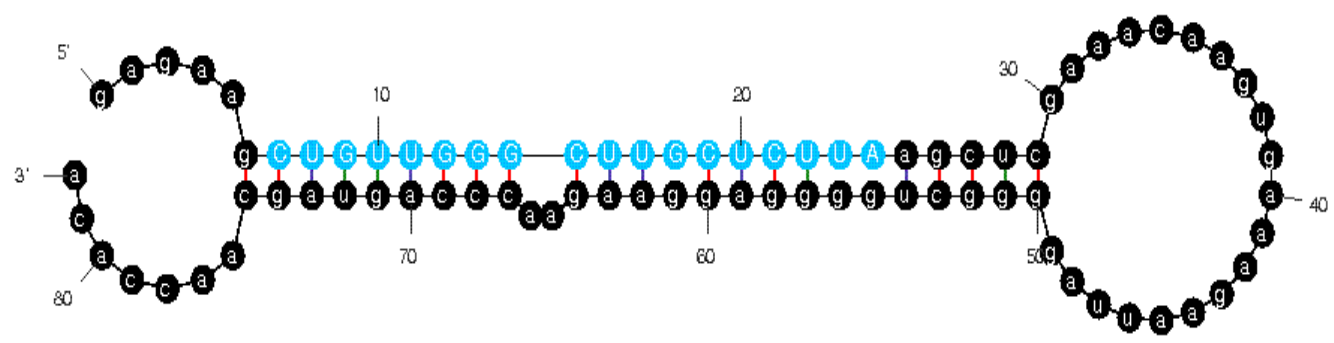

pmi-miRNew-20

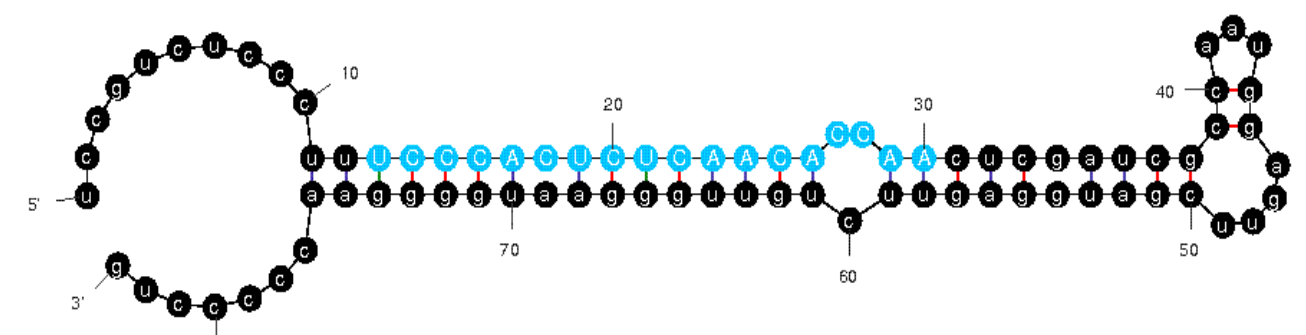

pmi-miRNew-21

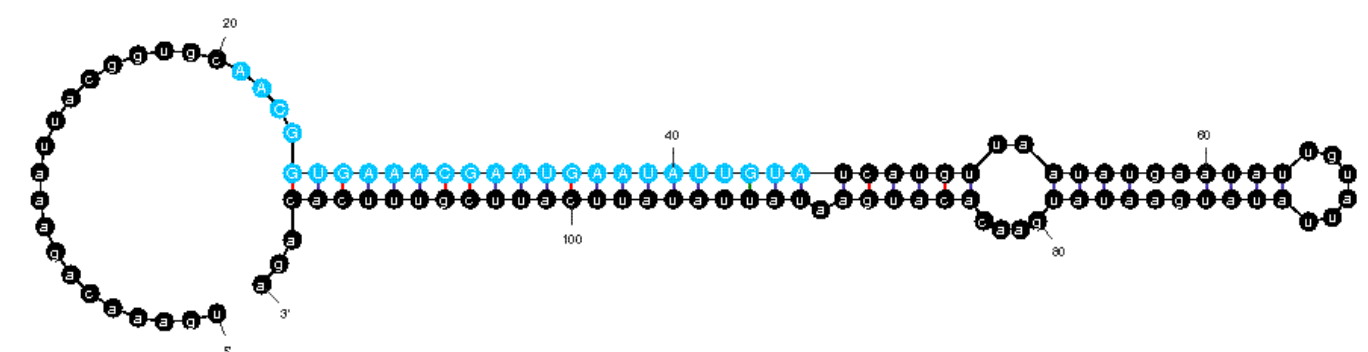

pmi-miRNew-22

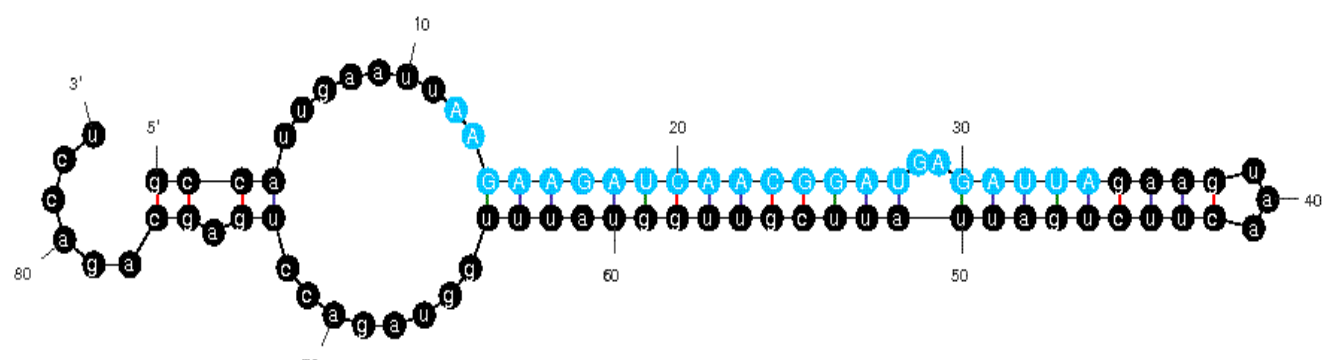


pmi-miRNew-23
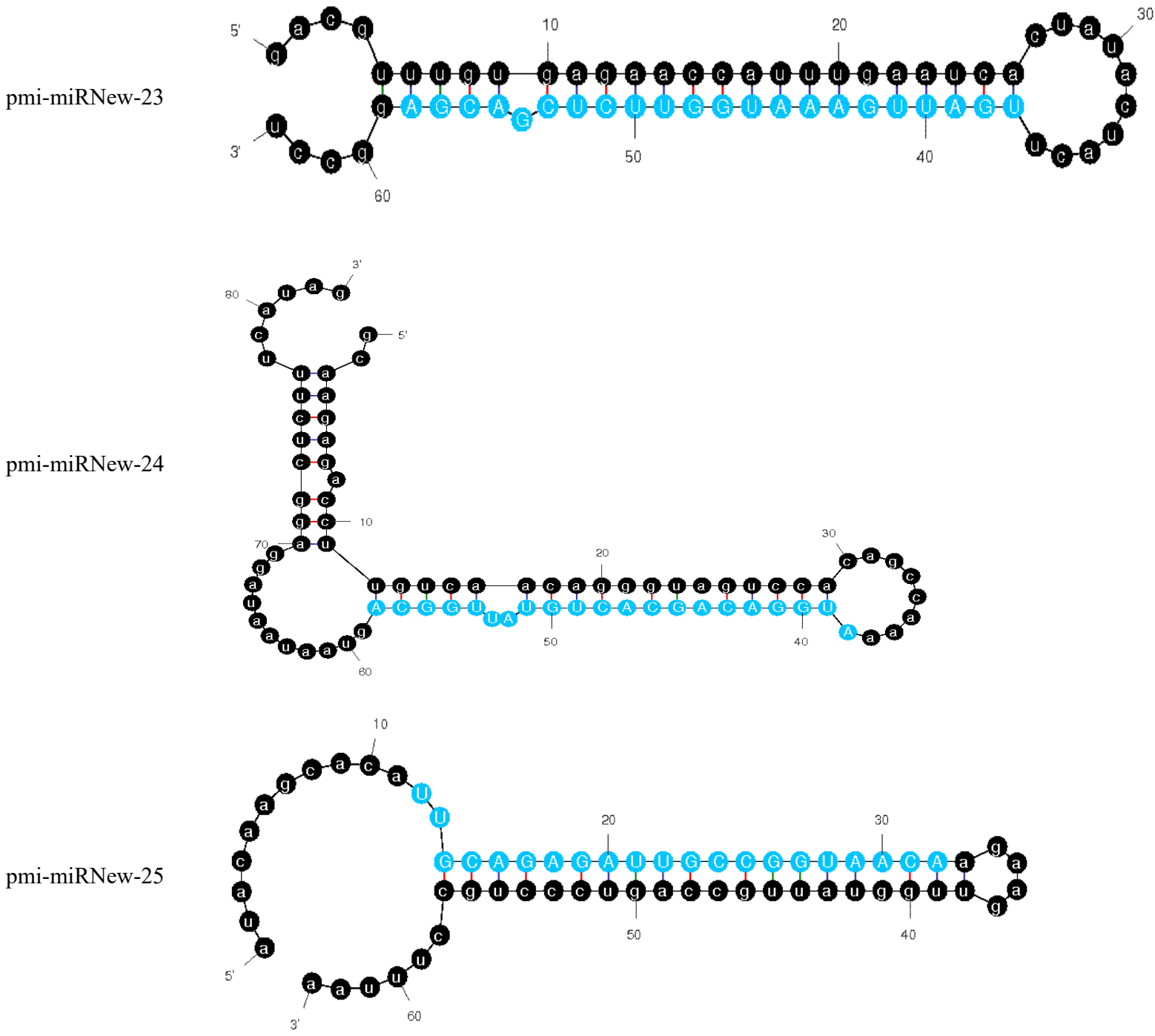

pmi-miRNew-26

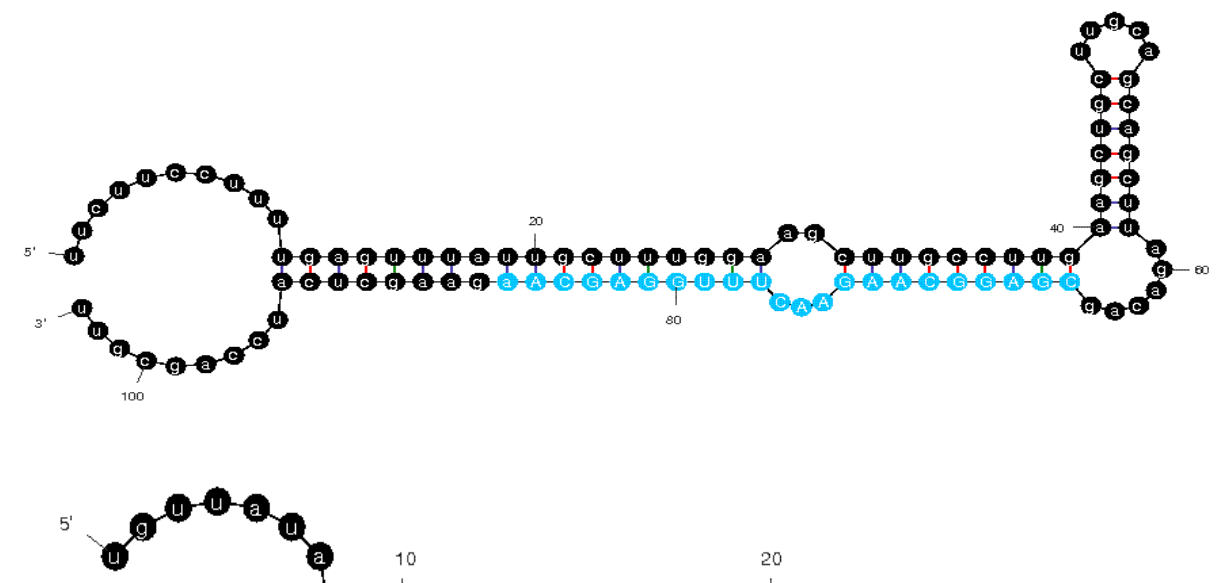

pmi-miRNew-27

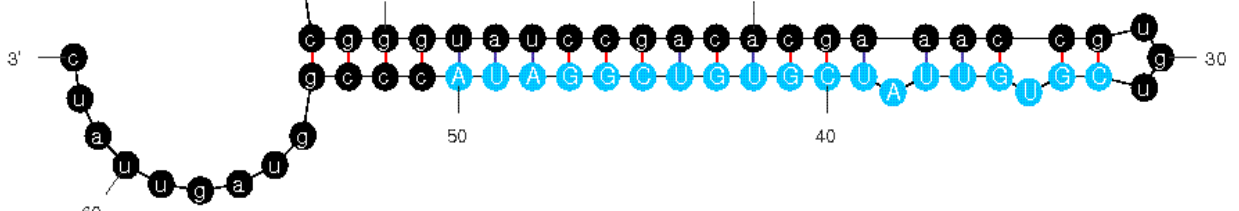


pmi-miRNew-28

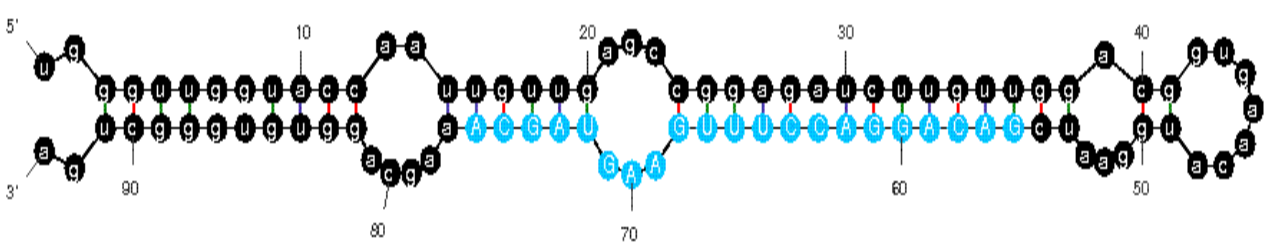

pmi-miRNew-29

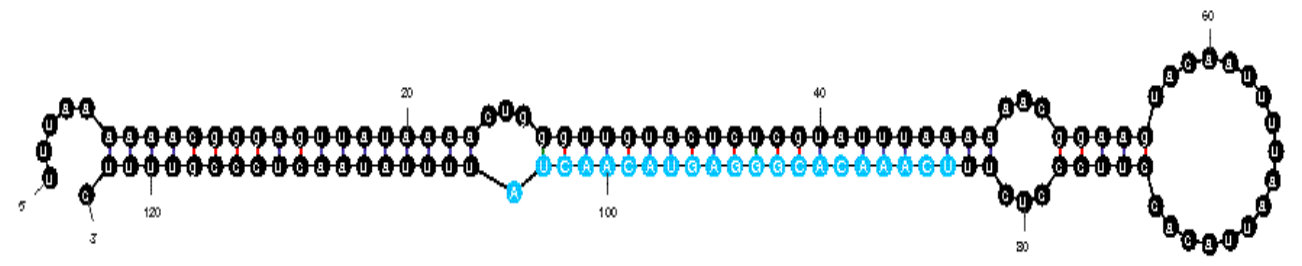

pmi-miRNew-29*

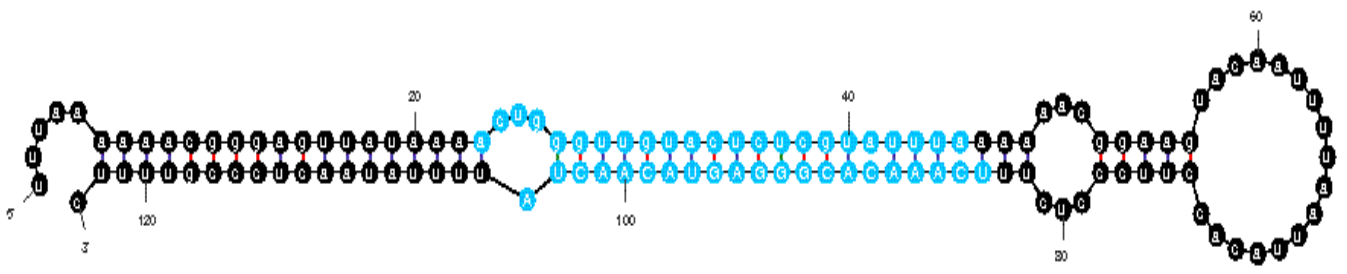

pmi-miRNew-30

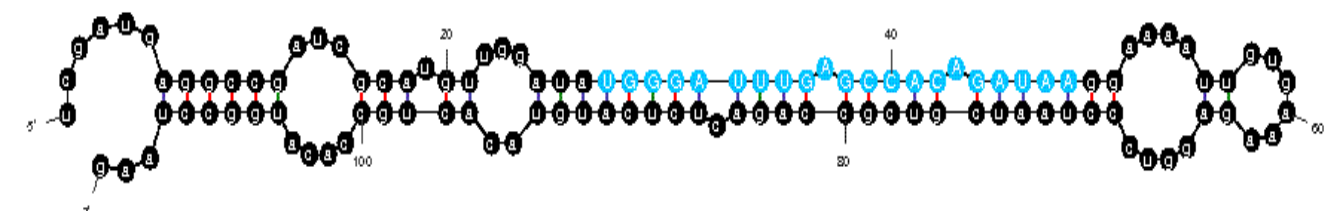

pmi-miRNew-31
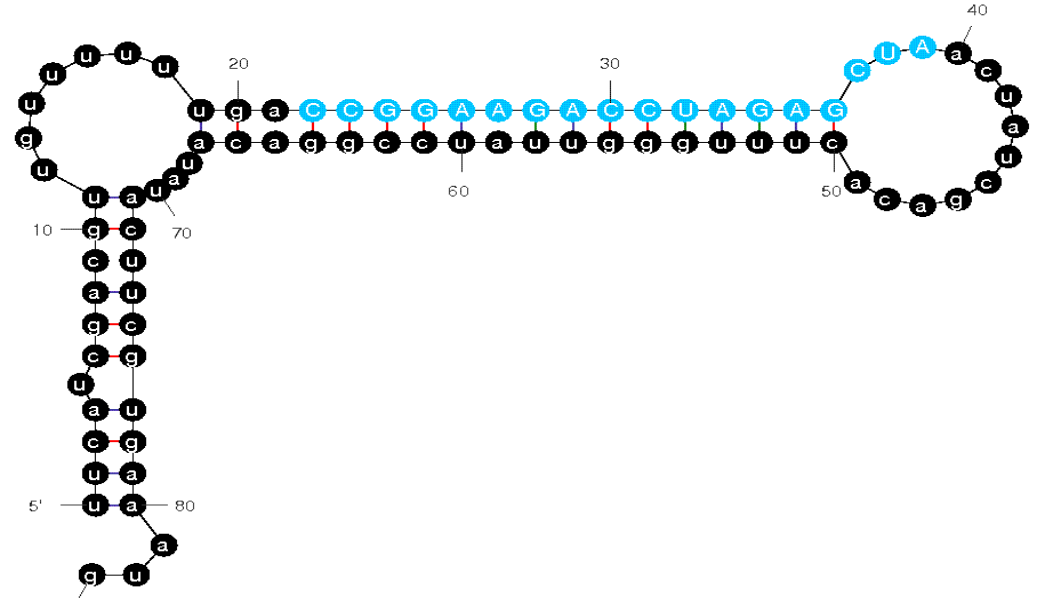

pmi-miRNew-32

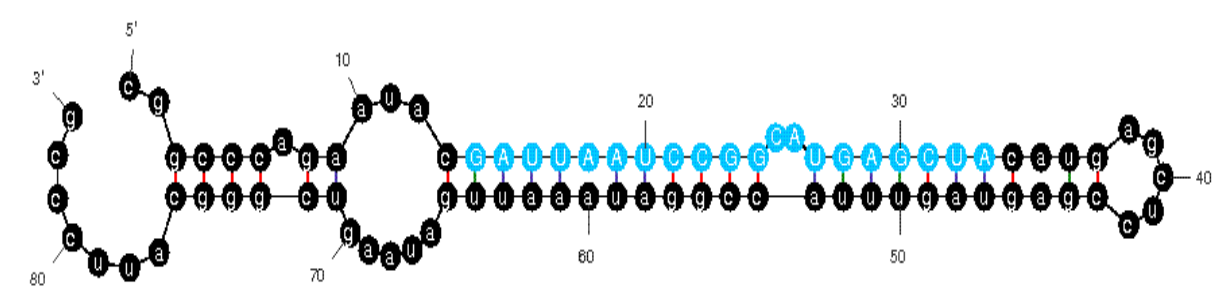


pmi-miRNew-33

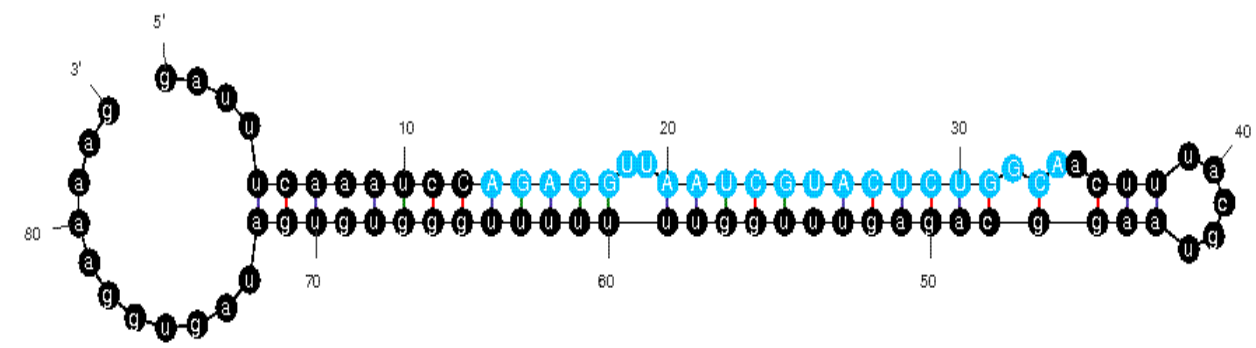

pmi-miRNew-34

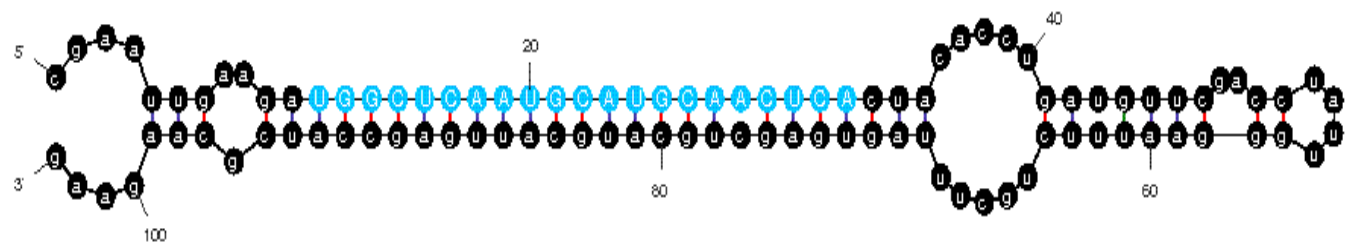

pmi-miRNew-34*

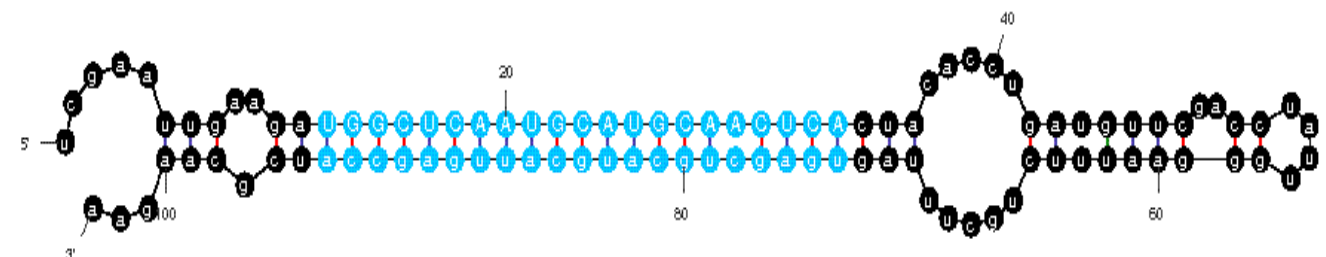

pmi-miRNew-35

pmi-miRNew-36
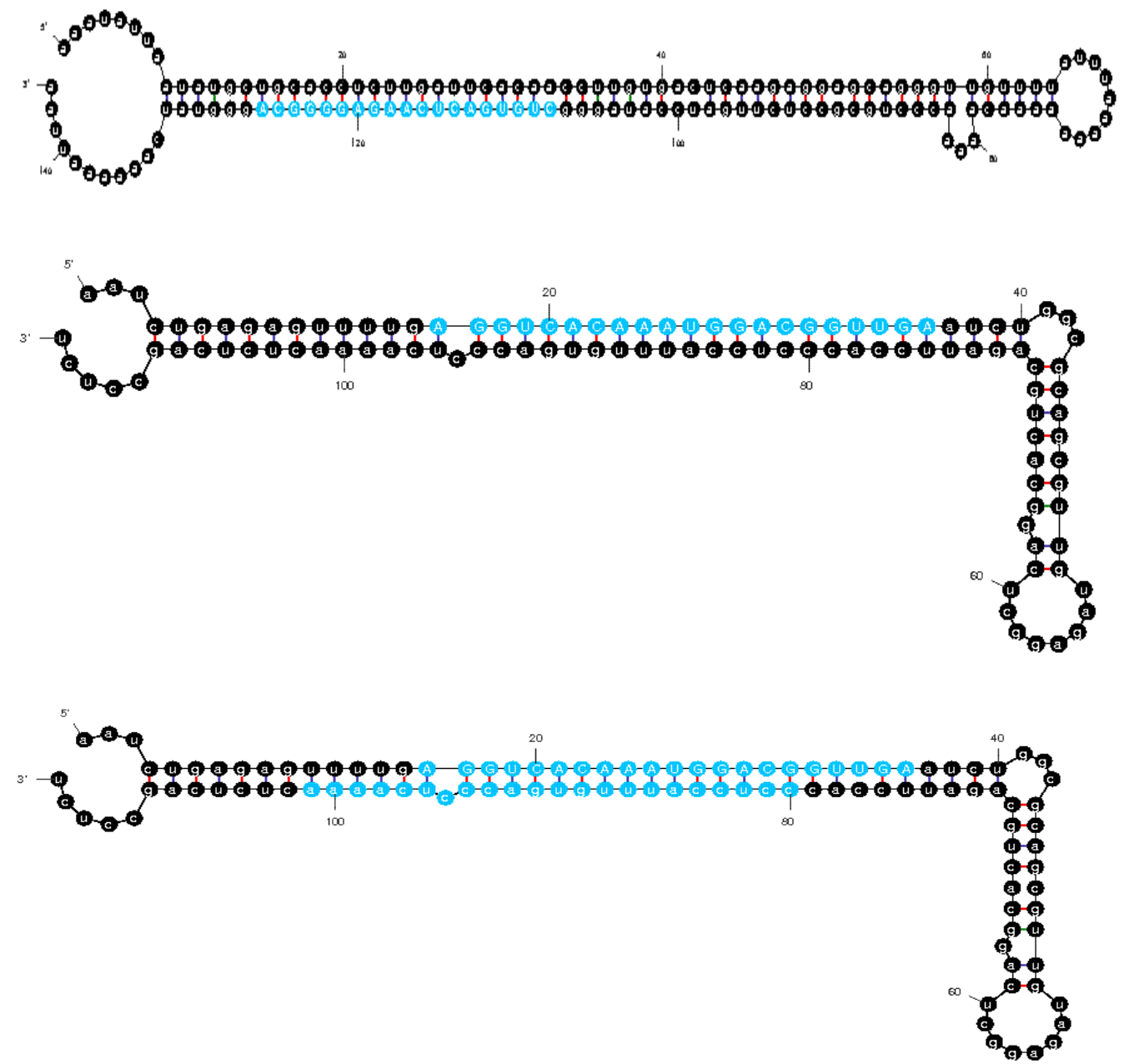

pmi-miRNew-36* 
pmi-miRNew-37

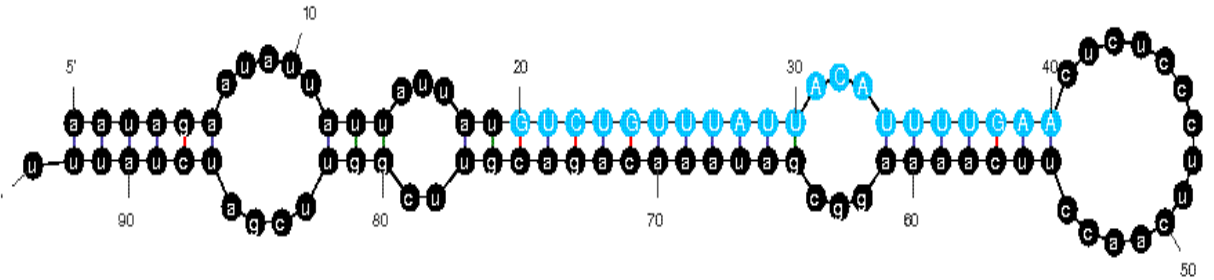

pmi-miRNew-38

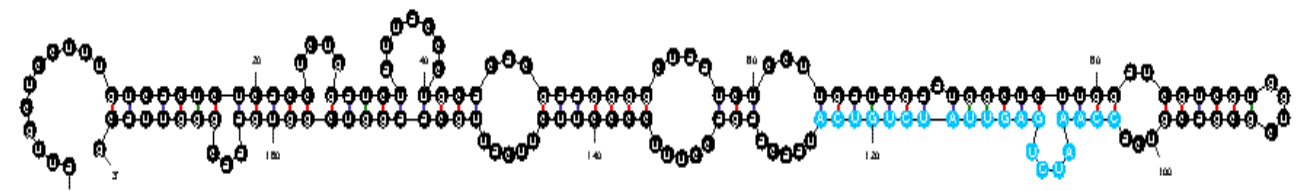

pmi-miRNew-39

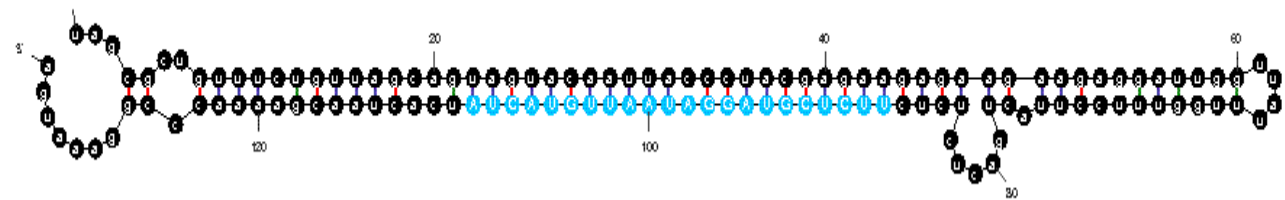

pmi-miRNew-39*

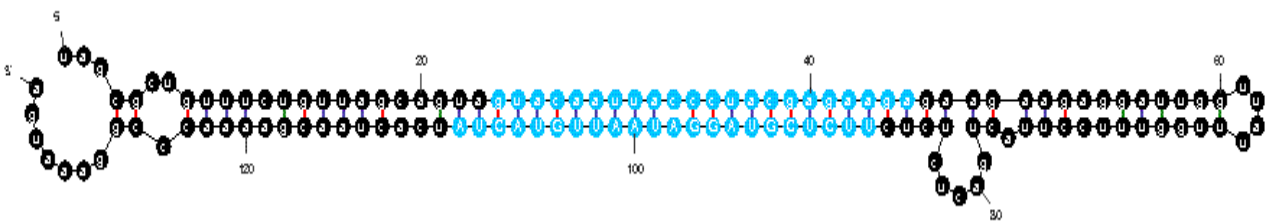

pmi-miRNew-40

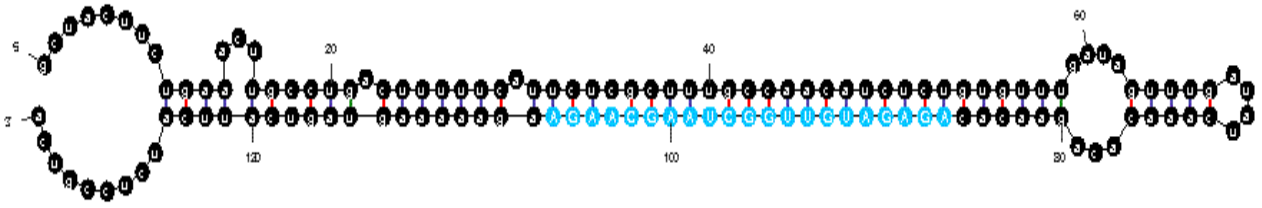

pmi-miRNew-41

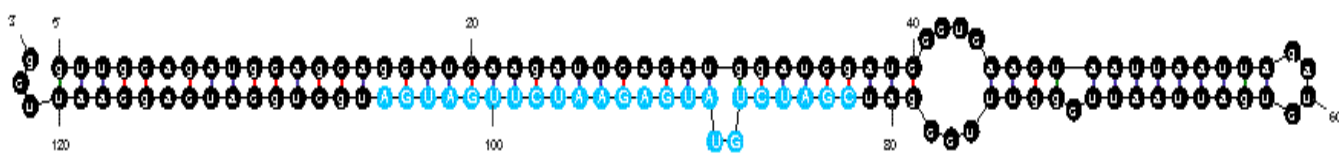

pmi-miRNew-42

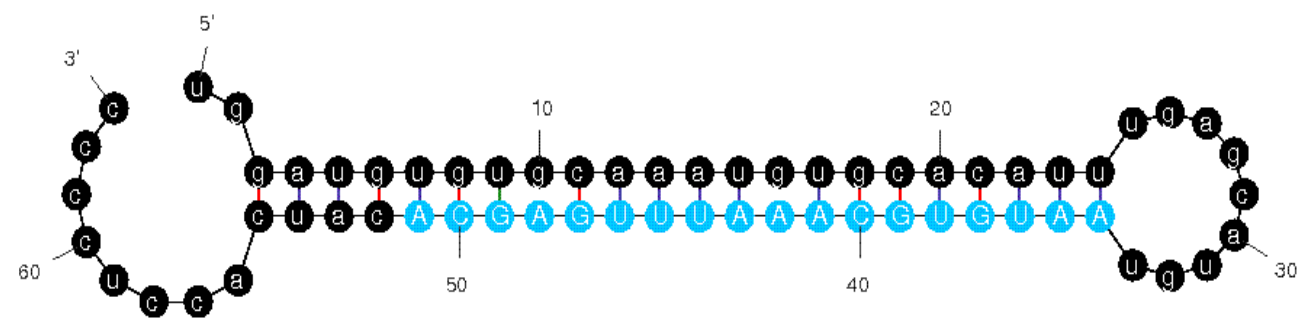


pmi-miRNew-43

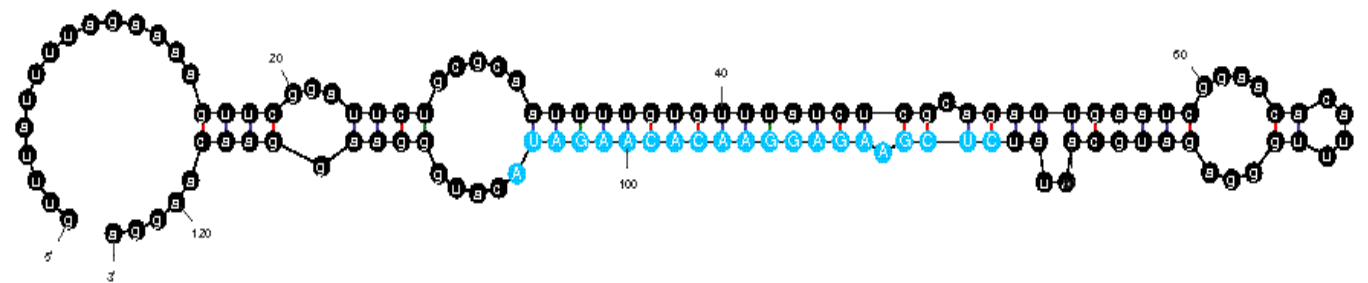

pmi-miRNew-44

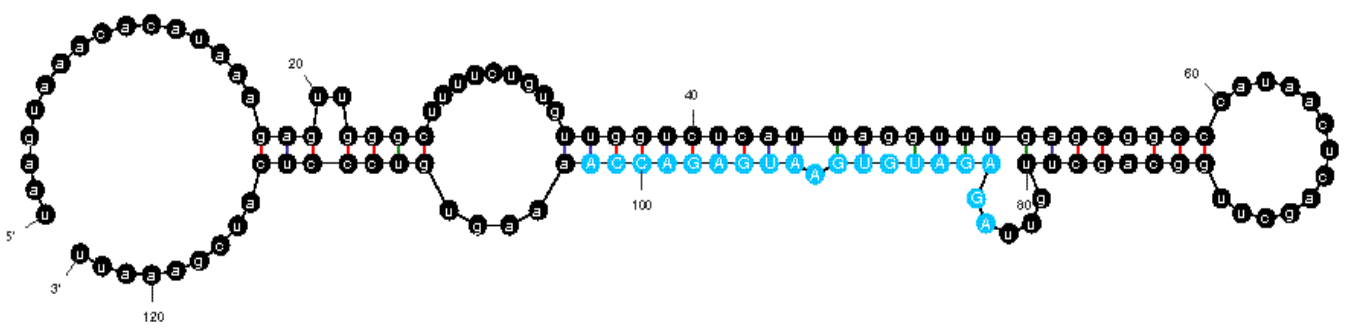

pmi-miRNew-45

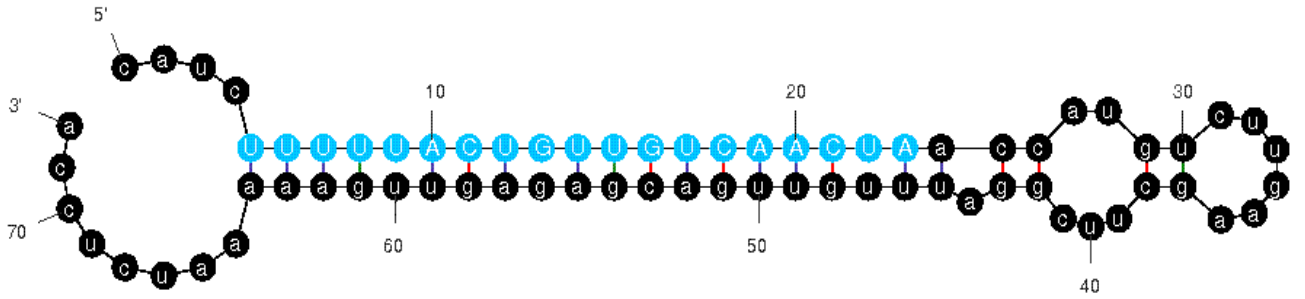

pmi-miRNew-46

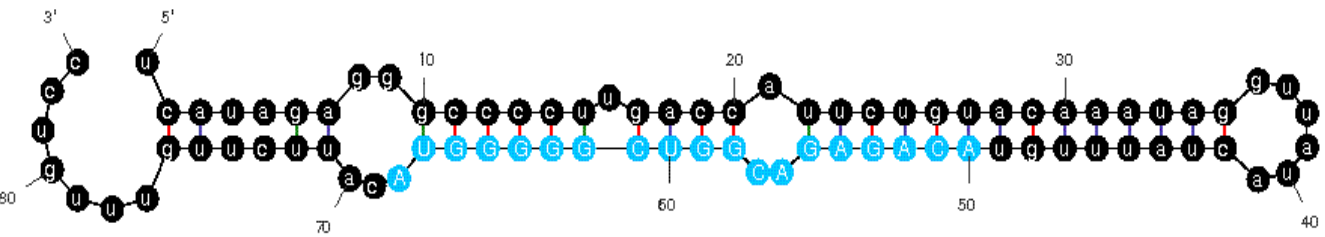

pmi-miRNew-46*

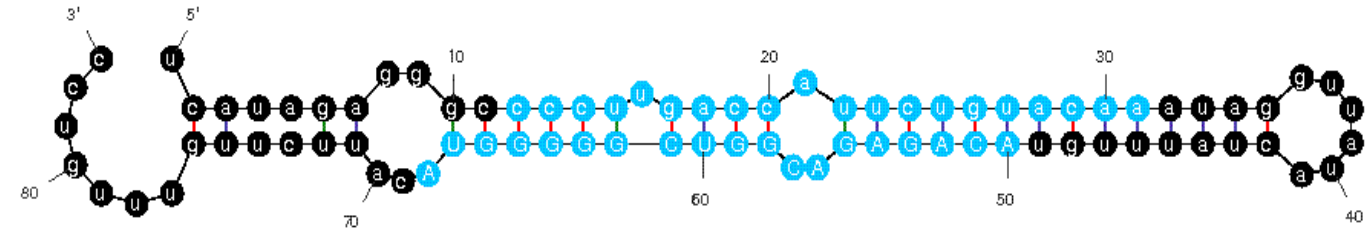

pmi-miRNew-47

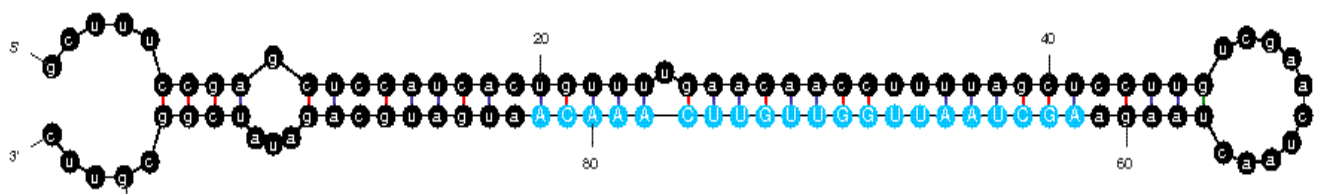


DIFFERENTIAL EXPRESSION OF MIRNA UNDER ABA AND MeJA TREATMENTS

Differential expression was carried out by comparing the normalized expression of miRNAs in the treatments (ABA and MeJA) against control libraries (K). In ABA treated plants, it was observed that 21 miRNAs were differentially regulated where two miRNAs were upregulated and 19 miRNAs were down-regulated. In MeJA treated plants, 38 miRNAs were differentially regulated which involved 24 up-regulated and 14 down-regulated miRNAs. This result demonstrated that majority of the miRNAs were more responsive towards MeJA (42\%) than ABA treatments (7\%) (Figure 3). Meanwhile, $51 \%$ of miRNA were significantly regulated in both libraries. All the significantly regulated miRNA were shown in Table 7.

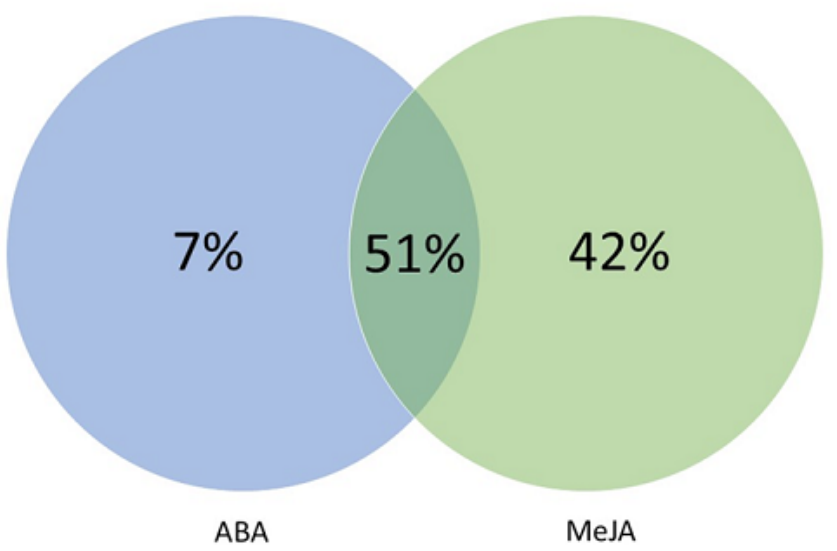

FIGURE 3. Venn diagram showing the common and specific sequence of significantly regulated miRNA in ABA and MeJA libraries

TABLE 7. List of significantly regulated miRNA under ABA and MeJA treatments. Negative and positive values indicated downand up-regulated expressions, respectively. Minus sign (-) indicated no miRNA expression detected in the particular library

\begin{tabular}{lccc}
\hline \multicolumn{1}{c}{ miRNA } & Mature sequence & \multicolumn{2}{c}{ Normalized Fold } \\
& & Change \\
\hline pmi-miR156d & GCTCTCTGTGCTTCTGTCGTCA & MeJA \\
pmi-miR156j & TTGACAGAAGAGAGTGAGTA & $-\infty .79$ \\
pmi-miR157d & TGCTCTCTGTGCTTCTGTCATCA & - & $\infty$ \\
pmi-miR159 & TTGGATTGAAGGGAGCTCTA & - & $\infty$ \\
pmi-miR159a & TTTGGATTGAAGGGAGCTCTACA & -9.70 & $\infty$ \\
pmi-miR160a & TGCCTGGCTCCCTGTATGCTTA & - & $\infty$ \\
pmi-miR162 & TCGATAAACCTCTGCATCCTA & - & $\infty$ \\
pmi-miR165a/b & TCGGACCAGGCTGCATCCCCA & $-\infty$ & - \\
pmi-miR166 & TCGGACCAGGCTTCATCCCA & $-\infty$ & - \\
pmi-miR166a & GAATGTTGTCTGGCTCGAGGA & $-\infty$ & $\infty$ \\
pmi-miR166b & CCGGACCAGGCCTCATTCCCCA & -6.40 & $-\infty$ \\
pmi-miR166c & TCGGACCAGGCTTCATTCCATA & $-\infty$ & - \\
pmi-miR166d & TCGTACCAGGCTTCATTCCCTA & $-\infty$ & $\infty$ \\
pmi-miR167a & TAAGCTGCCAGCATGATCGCA & - & $\infty$ \\
pmi-miR167b/d & TAAAGCTGCTAGCATGATCTGA & - & -13.58 \\
pmi-miR168 & TCGTTTGGTGCAGGTCGGGAA & - & $\infty$
\end{tabular}




\begin{tabular}{|c|c|c|c|}
\hline pmi-miR168b & CCCGCCTTGCACCAACTGAATA & - & $\infty$ \\
\hline pmi-miR169i/j/1 & TAGCCAAGGACGACTTGCCTGA & $-\infty$ & -5.06 \\
\hline pmi-miR172a & AGAATCTTGATGATGCTGCAGGA & - & $\infty$ \\
\hline pmi-miR319 & CTTGGACTGAAGGGAGCTCCTTA & -4.91 & -8.05 \\
\hline pmi-miR319b/d/e & TGGACTGAAGGGAGCTCCTA & $-\infty$ & -11.08 \\
\hline pmi-miR390 & CGCTATCTATCCTGAGCA & - & $-\infty$ \\
\hline pmi-miR393c & TCCAAAGGGATCGCATTGATTCA & - & $\infty$ \\
\hline pmi-miR396a & GTTCAATAAAGCTGTGGG & $-\infty$ & $-\infty$ \\
\hline pmi-miR396b & GTTCAATAAAGCTGTTGGAA & - & $\infty$ \\
\hline pmi-miR397a/b & TCATTGAGTGCAGCGTGGATGA & - & 7.78 \\
\hline pmi-miR398 & GGAGCGACCTGAGACCACATA & 4.46 & 3.72 \\
\hline pmi-miR398b & CGTGTTCGCAGGTCGCCCCTGA & $-\infty$ & $\infty$ \\
\hline pmi-miR399 & TGCCAAAGGAGAGTTGCCCTA & - & -6.24 \\
\hline pmi-miR408 & TGCACTGCCTCTTCCCTGGCAA & $-\infty$ & $\infty$ \\
\hline pmi-miR535 & TGACAATGAGAGAGAGCACTA & 5.36 & -8.05 \\
\hline pmi-miR535a & TGACAATGAGAGAGAGCACGT & - & -8.05 \\
\hline pmi-miR858 & TTCGTTGTCTGTTCAACCTTA & - & 9.03 \\
\hline pmi-miR894 & GATTCACGTCGGGTTCACCAA & -4.90 & 6.19 \\
\hline pmi-miR2916 & GGGGCTCGAAGACGATCAGATA & $-\infty$ & -4.08 \\
\hline pmi-miR4995 & AGGCAGTGGCTTGGTTAAGGA & -7.36 & -4.17 \\
\hline pmi-miR5077 & TCACGTCGGGTTCACCAG & - & 6.79 \\
\hline pmi-miR5368 & AGGGACAGTCTCAGGTAGACAGCA & - & 8.48 \\
\hline pmi-miR6173 & AGCCGTAAACGATGGATA & $-\infty$ & -16.30 \\
\hline pmi-miR6300 & GTCGTTGTAGTATAGTGGA & - & $-\infty$ \\
\hline pmi-miR6478 & CCGACCTTAGCTCAGTTGGTACA & $-\infty$ & $\infty$ \\
\hline
\end{tabular}

\section{ANALYSIS OF miRNA TARGET GENES}

miRNA function is closely related to its target gene. In this study, we employed psRNA Robot software to search for the miRNA targets. Table 8 showed a total of 37 potential target genes predicted in $P$. minor. Some miRNAs were identified to target the same genes (Table 8). Based on miRNA target prediction result, four miRNAs and targets were selected to be further explored due to their involvement in plant defense system and volatile compound biosynthesis pathway. The targets involved were peroxidase targeted by pmi-miR396a, 3-hydroxy3-methylglutaryl-CoA reductase (HMGR) targeted by pmi-miR6300, sesquiterpene synthase targeted by pmimiR6173 and alcohol dehydrogenase 1 (ADH1) targeted by pmi-miR396b. Additionally, analysis of target genes via gene ontology showed most of the targets belong to cellular component (35\%), followed by molecular function (34\%) and biological process (31\%) (Figure 4).

TABLE 8. List of predicted target genes

\begin{tabular}{rccc}
\hline miRNA & Score & ID transcript & Target annotation \\
\hline pmi-miR156d & 2.8 & comp53688_c0_seq1 & Photosystem II \\
pmi-miR156j & 2.8 & comp59110_c2_seq1 & Agrogenate dehydratase \\
& 2.2 & comp53137_c1_seq1 & SPL \\
pmi-miR157d & 3.0 & comp53825_c0_seq1 & F-box protein CPR30 \\
& 3.2 & comp59318_c1_seq1 & Probable ion channel POLLUX \\
& 3.8 & comp48954_c1_seq1 & 60S ribosomal protein L14-2
\end{tabular}




\begin{tabular}{|c|c|c|c|}
\hline \multirow[t]{2}{*}{ pmi-miR159 } & 1.0 & comp57600_c3_seq1 & Transcription factor GAMYB \\
\hline & 2.0 & comp67380_c0_seq2 & Putative disease resistance protein RGA3 \\
\hline \multirow[t]{2}{*}{ pmi-miR159a } & 1.0 & comp57600_c3_seq1 & Transcription factor GAMYB \\
\hline & 4.0 & comp10554_c0_seq1 & Growth-regulating factor 3 \\
\hline \multirow[t]{2}{*}{ pmi-miR160a } & 0.8 & comp55762_c0_seq1 & Auxin response factor \\
\hline & 3.5 & comp63097_c0_seq14 & Auxin-responsive protein IAA9 \\
\hline pmi-miR162 & N/A & N/A & N/A \\
\hline pmi-miR165a/b & 2.5 & comp52509_c0_seq3 & Wall-associated receptor kinase 2 \\
\hline \multirow[t]{2}{*}{ pmi-miR166 } & 0.2 & comp62172_c1_seq10 & Homeobox-leucine zipper protein HOX32 \\
\hline & 2.5 & comp67610_c2_seq1 & Probable WRKY transcription factor 19 \\
\hline pmi-miR166a & 0.0 & comp62276_c1_seq14 & $\begin{array}{l}\text { Vacuolar protein sorting-associated protein } \\
\qquad 35 \mathrm{~A}\end{array}$ \\
\hline pmi-miR166b & 2.5 & comp62172_c1_seq1 & Homeobox-leucine zipper protein HOX32 \\
\hline pmi-miR166c & 1.2 & comp62276_c1_seq14 & $\begin{array}{l}\text { Vacuolar protein sorting-associated protein } \\
\qquad 35 \mathrm{~A}\end{array}$ \\
\hline pmi-miR166d & 1.8 & comp62276_c1_seq14 & $\begin{array}{l}\text { Vacuolar protein sorting-associated protein } \\
\qquad 35 \mathrm{~A}\end{array}$ \\
\hline pmi-miR167a & 2.5 & comp64807_c0_seq2 & Putative $\mathrm{ABC}$ transporter $\mathrm{B}$ family member 8 \\
\hline pmi-miR167b/d & N/A & N/A & N/A \\
\hline pmi-miR168 & N/A & N/A & N/A \\
\hline pmi-miR168b & N/A & N/A & N/A \\
\hline pmi-miR169i/j/1 & 2.5 & comp67132_c1_seq1 & Protein MEI2-like2 \\
\hline pmi-miR172a & 1.5 & comp63292_c0_seq4 & Floral homeotic protein APETALA 2 \\
\hline pmi-miR319 & 2.5 & comp64847_c0_seq10 & Transcription factor GAMYB \\
\hline \multirow[t]{2}{*}{$\mathrm{pmi}-\mathrm{miR} 319 \mathrm{~b} / \mathrm{d} / \mathrm{e}$} & 2.0 & comp57600_c3_seq1 & Transcription factor GAMYB \\
\hline & 2.5 & comp50465_c0_seq1 & Transcription factor TC4 \\
\hline pmi-miR390 & 2.5 & comp53986_c0_seq1 & Cellulose synthase A catalytic subunit 6 \\
\hline pmi-miR393c & N/A & N/A & N/A \\
\hline pmi-miR396a & 2.5 & comp60490_c0_seq1 & Peroxidase \\
\hline pmi-miR396b & 2.5 & comp63431_c1_seq16 & ADH1 \\
\hline pmi-miR397a/b & 2.0 & comp67947_c0_seq1 & Laccase- 4 \\
\hline pmi-miR398 & 2.0 & comp61311_c1_seq2 & $\begin{array}{l}\text { Cytochrome c oxidase subunit } 5 \mathrm{~b}-2, \\
\text { mitochondrial }\end{array}$ \\
\hline pmi-miR398b & N/A & N/A & N/A \\
\hline pmi-miR399 & 2.0 & comp50399_c1_seq1 & Probable inorganic phosphate transporter \\
\hline pmi-miR408 & 2.2 & comp43803_c0_seq1 & Putative disease resistance protein At4g19050 \\
\hline pmi-miR535 & 2.5 & comp58725_c1_seq1 & GDP-mannose 3,5-epimerase 2 \\
\hline pmi-miR535a & 2.5 & comp58725_c1_seq1 & GDP-mannose 3,5-epimerase 2 \\
\hline pmi-miR858 & 2.0 & comp55943_c0_seq1 & 50S ribosomal protein L34 \\
\hline pmi-miR894 & N/A & N/A & N/A \\
\hline pmi-miR2916 & 1.8 & comp58044_c0_seq1 & Probable DNA primase large subunit \\
\hline pmi-miR4995 & 2.2 & comp62773_c1_seq3 & E3 ubiquitin ligase \\
\hline pmi-miR5077 & 2.5 & comp60152_c0_seq2 & Cell division protein FtsZ homolog 2-1 \\
\hline pmi-miR5368 & 1.0 & comp40772_c0_seq1 & Uncharacterized protein ORF91 \\
\hline pmi-miR6173 & 3.0 & comp46206_c0_seq1 & Probable sesquiterpene synthase \\
\hline \multirow[t]{2}{*}{ pmi-miR6300 } & 3.2 & comp55945_c0_seq1 & HMGR \\
\hline & 3.5 & comp59913_c0_seq1 & Proteasome subunit beta type-2-A \\
\hline pmi-miR6478 & $\mathrm{N} / \mathrm{A}$ & $\mathrm{N} / \mathrm{A}$ & N/A \\
\hline
\end{tabular}




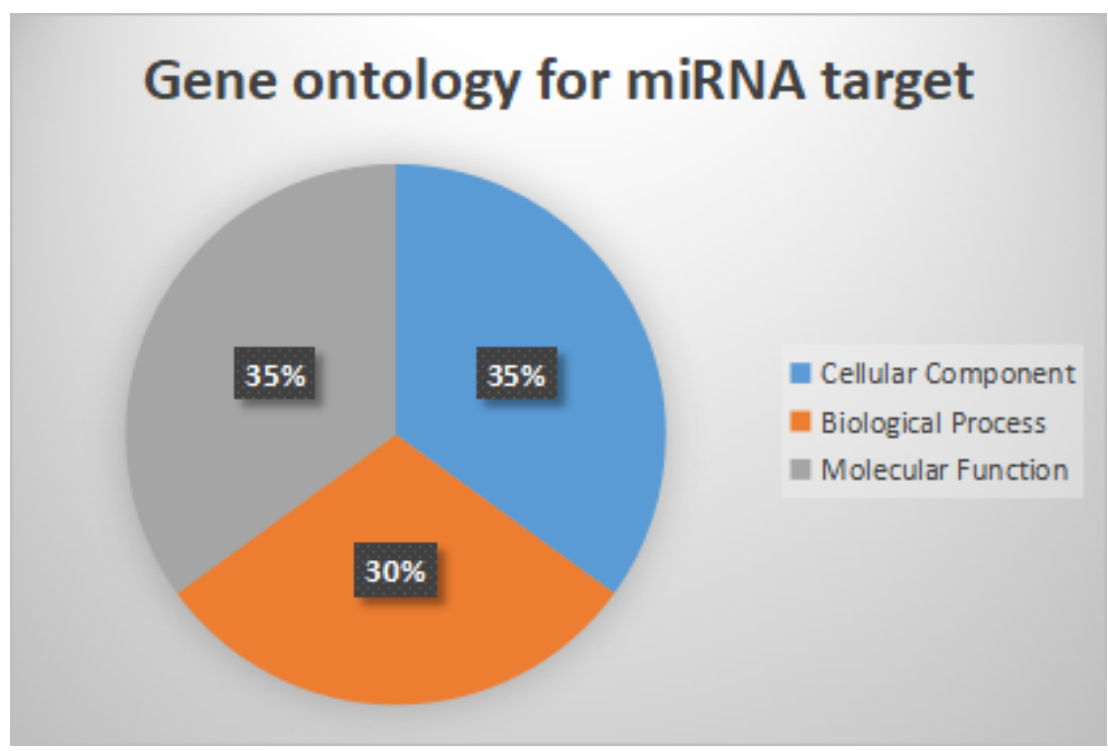

FIGURE 4. Pie chart showing the distribution of miRNA targets according to WEGO analysis

\section{EXPRESSION PROFILE OF SELECTED MIRNAS AND THEIR TARGETS USING RT-qPCR}

Four conserved miRNAs (pmi-miR396a, pmi-miR396b, pmi-miR6173, and pmi-miR6300) were selected for RTqPCR analysis. Based on high throughput sequencing, the selected miRNAs were detected in ABA and MeJA libraries except pmi-miR396b which was observed in MeJA library only. The RT-qPCR analysis was carried out to identify the expression of selected miRNAs throughout the treatments. The analysis results were shown in Figure 5 (A) and (B) for ABA and MeJA, respectively. Pmi-miR396a, pmi-miR6300 and pmiNew27 showed decreasing pattern in both ABA and MeJA treatments. Pmi-miR396b also showed similar pattern under MeJA treatments. In contrast, all the target genes showed increasing pattern. Pmi-miR6173 exhibited increasing pattern in ABA treatment while decreasing pattern under MeJA treatment. For its target genes, sesquiterpene synthase showed decreasing pattern under ABA treatment, while in MeJA treatment the expression was increased to two- fold and more than three-fold on Day 1 and Day 2, respectively. However, the expression of the target decreased on Day 3. In general, most of the miRNAs were down regulated under both treatments. In MeJA treatments, pmi-miR6173 and pmi-miR6300 had shown similar pattern (decreasing) with our previous study which involved $P$. minor treated with pathogenic fungi. In the study, both pmi-miR6173 and pmi-miR6300 were down regulated in Fusarium-treated compared to the control libraries (Samad et al. 2019). Our current study together with our recent study showed that the targets of pmi-miR6173 and pmi-miR6300 might play essential role in biotic and abiotic stresses in $P$. minor.
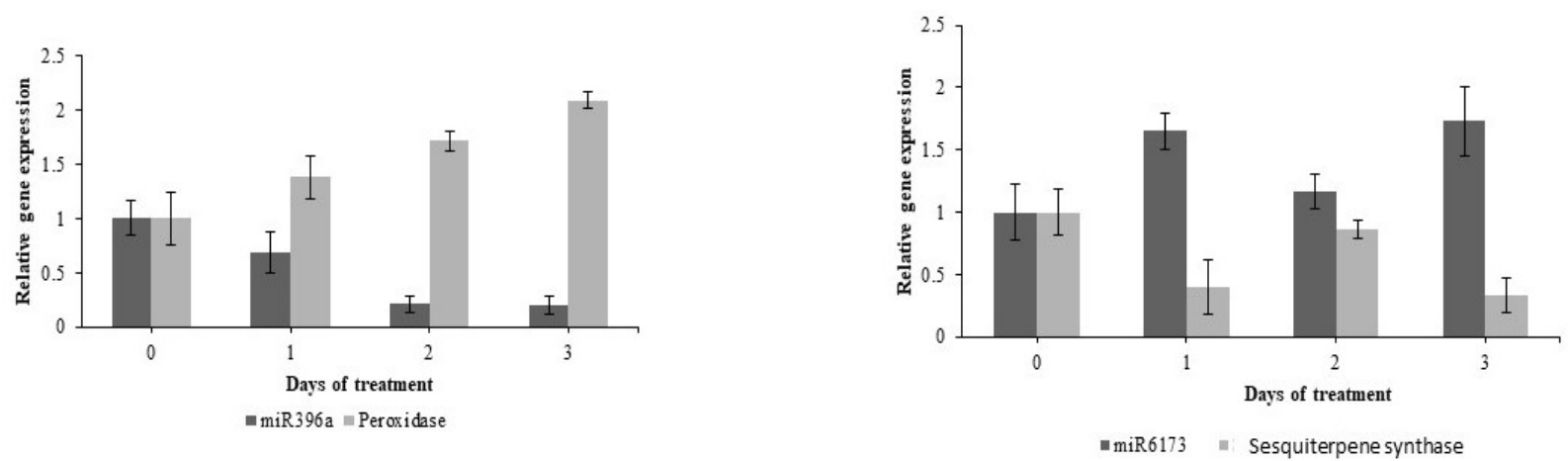


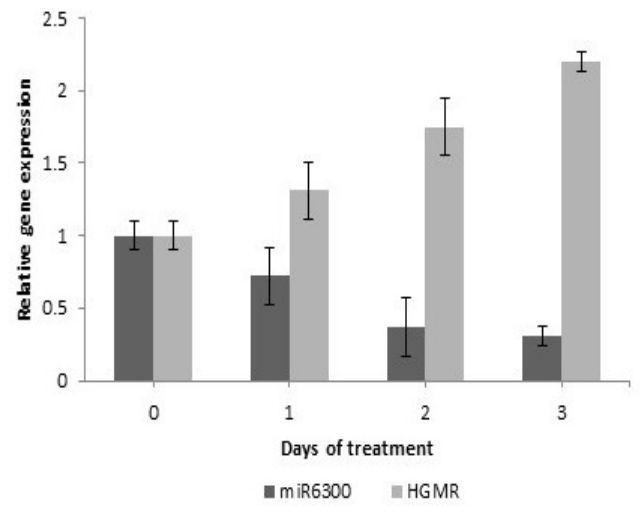

(A)
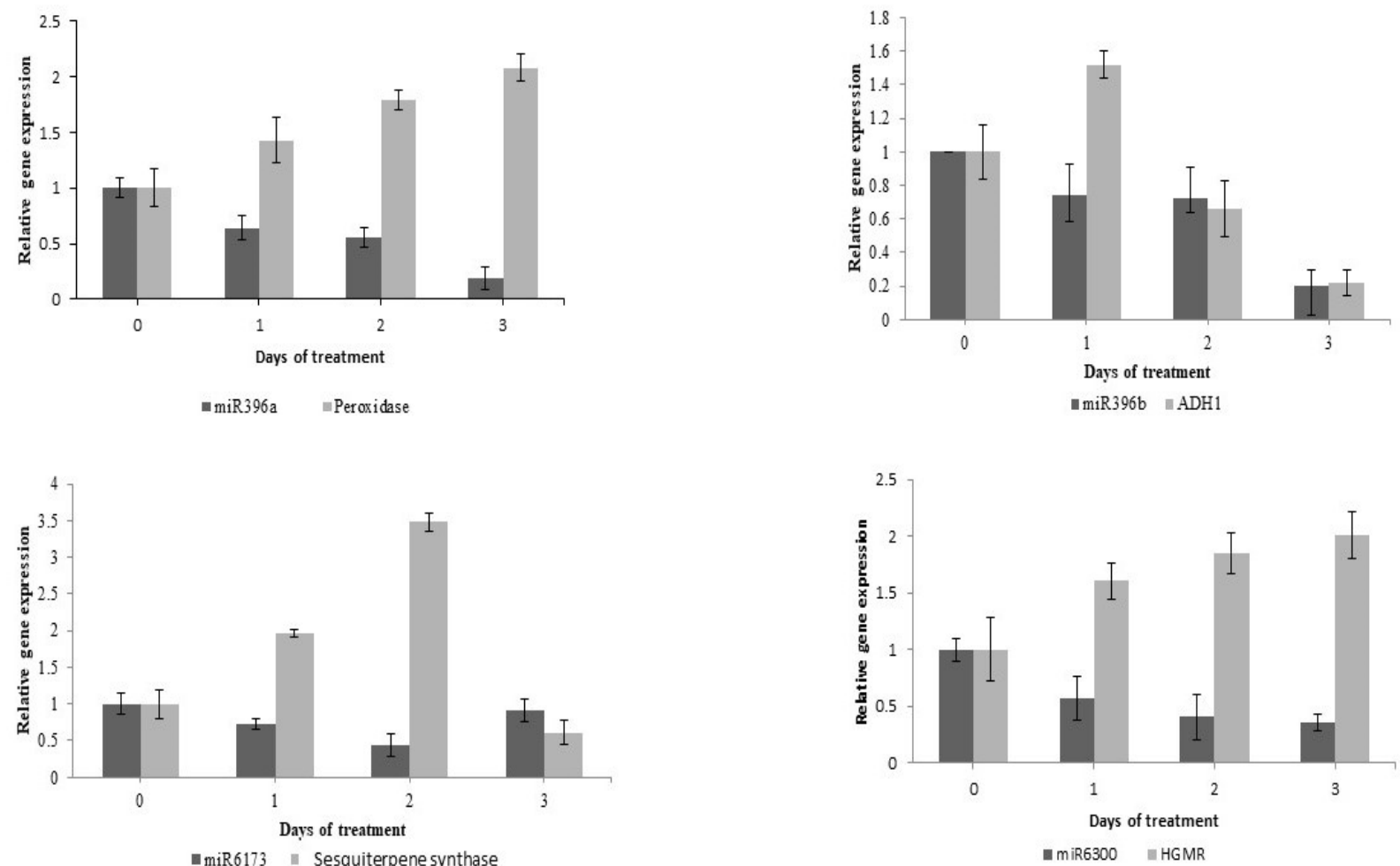

(B)

FIGURE 5. Relative expression of selected miRNAs and their targets in response to $\mathrm{ABA}(\mathrm{A})$ and $\mathrm{MeJA}(\mathrm{B})$ treatments

In this study, we observed that most miRNAs were being significantly regulated in MeJA than ABA libraries. This indicated that MeJA signaling pathway is way more diverse since previous studies revealed it could interact with other hormones, such as salicylic acid (SA), gibberellin (GA), ethylene (ET), auxin, 
brassinosteroid (BR) and even abscisic acid (ABA), to regulate gene expression in regulatory networks (Liu et al. 2015). These interactions led to major adjustments in plant biological processes including seed germination, root growth, flowering, senescence and stimulation of various secondary metabolite to counter insects and pathogen invasion (Huang et al. 2017). In contrast, ABA role is more focused in seed germination, stomatal closure and various abiotic stresses (Rai et al. 2011; Sah et al. 2016). In this study, we discovered a total of 41 conserved miRNAs that were responsive to ABA and MeJA treatments in $P$. minor. The targets involved were peroxidase targeted by pmi-miR396a, HMGR targeted by pmi-miR6300, sesquiterpene synthase targeted by pmimiR6173 and ADH1 targeted by pmi-miR396b. In ABA and MeJA treatments, the expression of miRNA and their targets were similar. These might happen because the crosstalk between the ABA and MeJA signaling pathways lead to similar changes in gene expression (Riemann et al. 2015). Previous findings showed both hormones contributed towards plant stress response by modulating the gene expression to synthesise secondary metabolites such as terpenoid indole alkaloid in Catharanthus roseus and anthocyanins in Arabidopsis thaliana (ElSayed \& Verpoorte 2004; Loreti et al. 2008). The target of miR396a, peroxidase, is an enzyme involved in cell elongation, lignification, seed germination, and defense response (Shigeto \& Tsutsumi 2016). The up-regulation of peroxidase is consistent with the ABA and MeJA roles as signal transduction pathway during plant stress (Almagro et al. 2009). High expression of peroxidase may induce the plant VOC as a response to the environmental stresses especially herbivore attack (War et al. 2011). The targets of pmi-miR6173 and pmi-miR6300, HMGR, and sesquiterpene synthase, respectively, are both involved in terpenoid biosynthesis pathway (Tholl 2015). HMGR is a rate limiting enzyme in MVA pathway which is required for accumulation of sesquiterpene (Chappell et al. 1991; Tholl 2015). In A. thaliana, loss of function for hmg 1 showed a $65 \%$ reduction in triterpene compound accumulation compared to the wild type (Ohyama et al. 2007). Moreover, mutant $h m g l$ also led to dwarfing, early senescence and male sterility, and reduced sterol levels (Suzuki et al. 2004). HMGR enzyme catalyses the conversion of HMG-CoA to mevalonate, which is later converted into mevalonate-5-phosphate through the enzyme MVK. High expression of HMGR gene induced by elicitor and wounding could enhance the sesquiterpene production (Chappell et al. 1991; Kondo et al. 2003). Similarly, sesquiterpene synthase is a type of terpene synthase required for sesquiterpene biosynthesis at downstream level (Tholl 2015). Functional analysis of $P$. minor sesquiterpene synthase led to the production of $\beta$-sesquiphellandrene in transgenic $A$. thaliana (Ee et al. 2014). Another study showed two novel sesquiterpene genes (PmSTPS1 and PmSTPS2) isolated from P. minor were responsible for the production of $\beta$-farnesene, $\alpha$-farnesene and farnesol. Additionally, PmSTPS 2 was found to produce nerolidol as an additional product compared to PmSTPS1 (Rusdi et al. 2018).

For pmi-miR396b target, ADH1 is involved in GLV biosynthesis pathway by catalysing the conversion of aldehydes to alcohols (Ul Hassan et al. 2015). GLVs have emerged as major players in plant defense, plantplant interactions and plant-insect interactions. Some GLVs inhibit the growth and proliferation of plant pathogens, including bacteria, fungi, and viruses. Furthermore, GLVs emitted from plants under herbivore attack can serve as aerial messengers to neighbouring plants and to attract parasitic or parasitoid enemies of the herbivores (Ul Hassan et al. 2015). In general, ADH are classified into two main superfamilies, mediumchain dehydrogenase/reductase (MDR) and short-chain dehydrogenase/reductase (SDR) which consist of 370 and 250 amino acid residues, respectively (Jörnvall 2008). In $P$. minor, this enzyme was reported to have two family members, PmADH1a and PmADH1b. Both of them were up-regulated under drought stress and involved in ABA signaling pathway (Abd Hamid et al. 2018).

\section{CONCLUSION}

High throughput sequencing and advance computational approaches have resulted in the accumulation of huge data on miRNAs. Therefore, exploration of miRNAs role in biological system becomes relatively easy than before. Investigation on miRNAs and their targets at each step of a particular pathway and identifying their significance are current approaches to decipher the functions of miRNAs in plant system. In this study, we managed to characterise miRNA in $P$. minor and their response under ABA and MeJA treatments. Four miRNAs related to volatile compound biosynthesis were selected to be further studied. However, lack of genome information resulted in the limitation of miRNA discovery in $P$. minor. We believe more miRNA related to various biological processes could be discovered with the availability of $P$. minor genome sequence. However, this study was essentially an attempt to provide the fundamental relationship between miRNAs and their response towards $\mathrm{ABA}$ and MeJA.

\section{ACKNOWLEDGEMENTS}

We would like to thank Institute of Bioscience, Universiti Putra Malaysia (UPM) and Malaysian Genome Institute (MGI) for providing the Bioanalyzer services. We also would like to extend our gratitude to anonymous reviewers for their comments on this manuscript. This work was funded by University Research Grant (DIP2015-018).

\section{REFERENCES}

Abd Hamid, N.A., Zainal, Z. \& Ismail, I. 2018. Two members of unassigned type of short-chain dehydrogenase/reductase superfamily (SDR) isolated from Persicaria minor show response towards ABA and drought stress. Journal of Plant 
Biochemistry and Biotechnology 27(3): 260-271.

Almagro, L., Gómez Ros, L.V., Belchi-Navarro, S., Bru, R., Ros Barceló, A. \& Pedreño, M.A. 2009. Class III peroxidases in plant defence reactions. Journal of Experimental Botany 60(2): 377-390.

Audic, S. \& Claverie, J.M. 1997. The significance of digital gene expression profiles. Genome Research 7(10): 986-995.

Baggerly, K.A., Deng, L., Morris, J.S. \& Aldaz, C.M. 2003. Differential expression in SAGE: Accounting for normal between-library variation. Bioinformatics 19(12): 1477-1483.

Baharum, S.N., Bunawan, H., Ghani, M.A., Mustapha, W.A. \& Noor, N.M. 2010. Analysis of the chemical composition of the essential oil of Polygonum minus Huds. using twodimensional gas chromatography-time-of-flight mass spectrometry (GC-TOF MS). Molecules 15(10): 7006-7015.

Benjamini, Y. \& Hochberg, Y. 1995. Controlling the false discovery rate: A practical and powerful approach to multiple testing. Journal of the Royal Statistical Society. Series B (Methodological) 57(1): 289-300.

Chappell, J., VonLanken, C. \& Vögeli, U. 1991. Elicitor-inducible 3-Hydroxy-3-Methylglutaryl coenzyme A reductase activity is required for sesquiterpene accumulation in tobacco cell suspension cultures. Plant Physiology 97(2): 693-698.

Christapher, P.V., Parasuraman, S., Christina, J.M., Asmawi, M.Z. \& Vikneswaran, M. 2015. Review on Polygonum minus. Huds, a commonly used food additive in Southeast Asia. Pharmacognosy Research 7(1): 1-6.

Ee, S.F., Mohamed-Hussein, Z.A., Othman, R., Shaharuddin, N.A., Ismail, I. \& Zainal, Z. 2014. Functional characterization of sesquiterpene synthase from Polygonum minus. The Scientific World Journal 2014: 840592.

El-Sayed, M. \& Verpoorte, R. 2004. Growth, metabolic profiling and enzymes activities of Catharanthus roseus seedlings treated with plant growth regulators. Plant Growth Regulation 44(1): 53-58.

Gor, M., Ismail, I., Mustapha, W., Zainal, Z., Noor, N., Othman, R. \& Hussein, Z. 2011. Identification of cDNAs for jasmonic acid-responsive genes in Polygonum minus roots by suppression subtractive hybridization. Acta Physiologiae Plantarum 33(2): 283-294.

Huang, H., Liu, B., Liu, L. \& Song, S. 2017. Jasmonate action in plant growth and development. Journal of Experimental Botany 68(6): 1349-1359.

Jörnvall, H. 2008. Medium- and short-chain dehydrogenase/ reductase gene and protein families: MDR and SDR gene and protein superfamilies. Cellular and Molecular Life Sciences: CMLS 65(24): 3873-3878

Kondo, K., Uritani, I. \& Oba, K. 2003. Induction mechanism of 3-hydroxy-3-methylglutaryl-CoA reductase in potato tuber and sweet potato root tissues. Bioscience, Biotechnology and Biochemistry 67(5): 1007-1017.

Kozomara, A., Birgaoanu, M. \& Griffiths-Jones, S. 2019. miRBase: From microRNA sequences to function. Nucleic Acids Research 47(D1): D155-D162.

Lenka, S.K., Nims, N.E., Vongpaseuth, K., Boshar, R.A., Roberts, S.C. \& Walker, E.L. 2015. Jasmonate-responsive expression of paclitaxel biosynthesis genes in Taxus cuspidata cultured cells is negatively regulated by the bHLH transcription factors TcJAMYC1, TcJAMYC2, and TcJAMYC4. Frontiers in Plant Science 6: 115-115.

Liang, Z., Ma, Y., Xu, T., Cui, B., Liu, Y., Guo, Z. \& Yang, D. 2013. Effects of abscisic acid, gibberellin, ethylene and their interactions on production of phenolic acids in Salvia miltiorrhiza bunge hairy roots. PLoS ONE 8(9): e72806.

Liu, Z., Zhang, S., Sun, N., Liu, H., Zhao, Y., Liang, Y., Zhang, L. \& Han, Y. 2015. Functional diversity of jasmonates in rice. Rice (N Y) 8(1): 42.

Livak, K.J. \& Schmittgen, T.D. 2001. Analysis of relative gene expression data using real-time quantitative PCR and the 2- $\Delta \Delta C$ T method. Methods 25(4): 402-408.

Loke, K.K., Rahnamaie-Tajadod, R., Yeoh, C.C., Goh, H.H., Mohamed-Hussein, Z.A., Mohd Noor, N., Zainal, Z. \& Ismail, I. 2016. RNA-seq analysis for secondary metabolite pathway gene discovery in Polygonum minus. Genomics Data 7: 12-13.

Loreti, E., Povero, G., Novi, G., Solfanelli, C., Alpi, A. \& Perata, P. 2008. Gibberellins, jasmonate and abscisic acid modulate the sucrose-induced expression of anthocyanin biosynthetic genes in Arabidopsis. New Phytologist 179(4): 1004-1016.

Markham, N.R. \& Zuker, M. 2008. UNAFold: Software for nucleic acid folding and hybridization. Methods in Molecular Biology 453: 3-31.

Nazaruddin, N., Samad, A.F.A., Sajad, M., Jani, J., Zainal, Z. \& Ismail, I. 2017. Small RNA-seq analysis in response to methyl jasmonate and abscisic acid treatment in Persicaria minor. Genomics Data 12: 157-158.

Ohyama, K., Suzuki, M., Masuda, K., Yoshida, S. \& Muranaka, T. 2007. Chemical phenotypes of the $h m g 1$ and $h m g 2$ mutants of Arabidopsis demonstrate the In-planta role of HMG-CoA reductase in triterpene biosynthesis. Chemical and Pharmaceutical Bulletin (Tokyo) 55(10): 1518-1521.

Pontes, O., Costa-Nunes, P., Vithayathil, P. \& Pikaard, C.S. 2009. RNA polymerase V functions in Arabidopsis interphase heterochromatin organization independently of the 24-nt siRNA-directed DNA methylation pathway. Molecular Plant 2(4): 700-710.

Rahnamaie-Tajadod, R., Loke, K.K., Goh, H.H. \& Mohd Noor, N. 2017. Differential gene expression analysis in Polygonum minus leaf upon 24 hours of methyl jasmonate elicitation. Frontiers in Plant Science 8(109). doi: 10.3389/ fpls.2017.00109.

Rai, M.K., Shekhawat, N.S., Harish, Gupta, A.K., Phulwaria, M., Ram, K. \& Jaiswal, U. 2011. The role of abscisic acid in plant tissue culture: a review of recent progress. Plant Cell, Tissue and Organ Culture (PCTOC) 106(2): 179-190.

Riemann, M., Dhakarey, R., Hazman, M., Miro, B., Kohli, A. \& Nick, P. 2015. Exploring jasmonates in the hormonal network of drought and salinity responses. Frontiers in Plant Science 6: 1077.

Roslan, N.D., Yusop, J.M., Baharum, S.N., Othman, R., Mohamed-Hussein, Z.A., Ismail, I., Noor, N.M. \& Zainal, Z. 2012. Flavonoid biosynthesis genes putatively identified in the aromatic plant Polygonum minus via Expressed Sequences Tag (EST) analysis. International Journal of Molecular Sciences 13(3): 2692-2706.

Rusdi, N.A., Goh, H.H., Sabri, S., Ramzi, A.B., Mohd Noor, N. \& Baharum, S.N. 2018. Functional characterisation of new sesquiterpene synthase from the Malaysian herbal plant, Polygonum minus. Molecules (Basel, Switzerland) 23(6): 1370.

Sah, S.K., Reddy, K.R. \& Li, J. 2016. Abscisic acid and abiotic stress tolerance in crop plants. Frontiers in Plant Science 7: $571-571$. 
Samad, A.F.A., Rahnamaie-Tajadod, R., Sajad, M., Jani, J., Murad, A.M.A., Noor, N.M. \& Ismail, I. 2019. Regulation of terpenoid biosynthesis by miRNA in Persicaria minor induced by Fusarium oxysporum. BMC Genomics 20(1): 586.

Samad, A.F.A., Nazaruddin, N., Murad, A.M.A., Jani, J., Zainal, Z. \& Ismail, I. 2018. Deep sequencing and in silico analysis of small RNA library reveals novel miRNA from leaf Persicaria minor transcriptome. 3 Biotech 8(3): 136.

Samad, A.F.A., Sajad, M., Nazaruddin, N., Fauzi, I.A., Murad, A.M.A., Zainal, Z. \& Ismail, I. 2017. MicroRNA and transcription factor: Key players in plant regulatory network. Frontiers in Plant Science 8: 565.

Shigeto, J. \& Tsutsumi, Y. 2016. Diverse functions and reactions of class III peroxidases. New Phytologist 209(4): 1395-1402.

Suzuki, M., Kamide, Y., Nagata, N., Seki, H., Ohyama, K., Kato, H., Masuda, K., Sato, S., Kato, T., Tabata, S., Yoshida, S. \& Muranaka, T. 2004. Loss of function of 3-hydroxy3-methylglutaryl coenzyme A reductase 1 (HMG1) in Arabidopsis leads to dwarfing, early senescence and male sterility, and reduced sterol levels. The Plant Journal 37(5): 750-761.

Tholl, D. 2015. Biosynthesis and biological functions of terpenoids in plants. Advances in Biochemical Engineering/ Biotechnology 148: 63-106.

U1 Hassan, M.N., Zainal, Z. \& Ismail, I. 2015. Green leaf volatiles: Biosynthesis, biological functions and their applications in biotechnology. Plant Biotechnology Journal 13(6): 727-739.

War, A.R., Sharma, H.C., Paulraj, M.G., War, M.Y. \& Ignacimuthu, S. 2011. Herbivore induced plant volatiles: Their role in plant defense for pest management. Plant Signaling \& Behavior 6(12): 1973-1978.

Wu, H.J., Ma, Y.K., Chen, T., Wang, M. \& Wang, X.J. 2012. PsRobot: A web-based plant small RNA meta-analysis toolbox. Nucleic Acids Research 40: 22-28.

Yan, C. \& Xie, D. 2015. Jasmonate in plant defence: Sentinel or double agent? Plant Biotechnology Journal 13(9): 12331240.

Yang, D., Ma, P., Liang, X., Wei, Z., Liang, Z., Liu, Y. \& Liu, F. 2012. PEG and ABA trigger methyl jasmonate accumulation to induce the MEP pathway and increase tanshinone production in Salvia miltiorrhiza hairy roots. Physiologia Plantarum 146(2): 173-183.
Ye, J., Zhang, Y., Cui, H., Liu, J., Wu, Y., Cheng, Y., Xu, H., Huang, X., Li, S., Zhou, A., Zhang, X., Bolund, L., Chen, Q., Wang, J., Yang, H., Fang, L. \& Shi, C. 2018. WEGO 2.0: A web tool for analyzing and plotting GO annotations, 2018 update. Nucleic Acids Research 46(W1): W71-W75.

Zhang, B.H., Pan, X.P., Cox, S.B., Cobb, G.P. \& Anderson, T.A. 2006. Evidence that miRNAs are different from other RNAs. Cellular and Molecular Life Sciences CMLS 63(2): 246-254.

Zhang, B., Pan, X. \& Stellwag, E.J. 2008. Identification of soybean microRNAs and their targets. Planta 229(1): 161182.

Abdul Fatah A. Samad \& Ismanizan Ismail* Institute of Systems Biology

Universiti Kebangsaan Malaysia

43600 UKM Bangi, Selangor Darul Ehsan

Malaysia

Abdul Fatah A. Samad

Department of Biosciences

Faculty of Science

Universiti Teknologi Malaysia

81310 UTM Skudai, Johor Darul Takzim

Malaysia

Nazaruddin Nazaruddin \& Jaeyres Jani

Department of Chemistry

Faculty of Mathematics and Natural Sciences

Syiah Kuala University

Darussalam, 23111 Banda Aceh

Indonesia

Ismanizan Ismail*

Centre for Biotechnology and Functional Food

Faculty of Science and Technology

Universiti Kebangsaan Malaysia

43600 UKM Bangi, Selangor Darul Ehsan

Malaysia

*Corresponding author; email: maniz@ukm.edu.my

Received: 9 August 2019

Accepted: 14 February 2020 